ESAIM: M2AN 48 (2014) 1089-1116

DOI: $10.1051 / \mathrm{m} 2 \mathrm{an} / 2013132$
ESAIM: Mathematical Modelling and Numerical Analysis

www.esaim-m2an.org

\title{
A REDUCED MODEL FOR DARCY'S PROBLEM IN NETWORKS OF FRACTURES *
}

\author{
Luca Formaggia ${ }^{1}$, Alessio Fumagalli ${ }^{1,2}$, Anna Scotti $^{1}$ and Paolo Ruffo ${ }^{3}$
}

\begin{abstract}
Subsurface flows are influenced by the presence of faults and large fractures which act as preferential paths or barriers for the flow. In literature models were proposed to handle fractures in a porous medium as objects of codimension 1. In this work we consider the case of a network of intersecting fractures, with the aim of deriving physically consistent and effective interface conditions to impose at the intersection between fractures. This new model accounts for the angle between fractures at the intersections and allows for jumps of pressure across intersections. This fact permits to describe the flow when fractures are characterized by different properties more accurately with respect to other models that impose pressure continuity. The main mathematical properties of the model, derived in the two-dimensional setting, are analyzed. As concerns the numerical discretization we allow the grids of the fractures to be independent, thus in general non-matching at the intersection, by means of the extended finite element method (XFEM). This increases the flexibility of the method in the case of complex geometries characterized by a high number of fractures.
\end{abstract}

Mathematics Subject Classification. 65N30, 76S05, 86A60.

Received February 12, 2013. Revised September 18, 2013.

Published online July 2, 2014.

\section{INTRODUCTION}

The presence of fractures can largely influence the flow in porous media in geophysical applications. The literature on flow in fractured media is rather extensive, we give here just some main references $[2,3,8,10]$ and we mention the applications to reservoir simulations $[21,26,28]$.

Fractured rocks are characterized by fractures covering a large range of space scales, from small joints to large faults. In particular, large fractures and faults can act, according to their different permeability, as barriers or

Keywords and phrases. Reduced models, fractured porous media, XFEM.

* This work has been supported by ENI s.p.a. The first and third authors also acknowledge the support of MIUR through the PRIN09 project n. 2009 Y 4 R $3 B$ B 001.

1 MOX - Dipartimento di Matematica "F. Brioschi" - Politecnico di Milano - via Bonardi 9, 20133 Milan, Italy.

luca.formaggia@polimi.it; anna.scotti@polimi.it

2 IFP Energies nouvelles -1 and 4, avenue de Bois-Prau, 92852 Rueil-Malmaison Cedex, France.

alessio.fumagalli1984@gmail.com

3 ENI Spa - Exploration and Production Division - 5 Palazzo Uffici, Room 4046 E, GEBA Dept. - via Emilia 1, San Donato Milanese, 20097 (MI), Italy. paolo.ruffo@eni.com 
preferential paths to the flow. This is due to the fact that fractures may be produced at different geological times and may undergo infilling and other geochemical processes that may modify their permeability considerably [17].

At a different space scale, micro fractures can alter, according to their density and orientation, the overall permeability of the porous medium. Numerical simulations of problems related to groundwater flow such as $\mathrm{CO}_{2}$ storage, oil migration and recovery or groundwater contamination should be able to account for the presence of fractures to yield accurate results.

In the applications we are considering, the porous medium is usually characterized by the presence of several fractures that can intersect each other. Moreover the characteristic thickness, or aperture, of the fractures is very small compared to their length and, in particular, compared to the typical size of the domain of interest. This geometric complexity makes the simulations particularly challenging for standard methods.

In $[5,24,27]$ the authors propose a model reduction strategy to overcome part of the aforementioned problems by using a domain decomposition approach where fractures are represented as one-codimension interfaces inside the porous domains. The proposed model can successfully reduce the number of unknowns in the simulation since, instead of refining the grid to capture a thin $n$-dimensional region we are replacing it with a $n$-1-dimensional interface. This approach, originally developed for single-phase Darcy problems, has been successfully extended to passive transport in porous media [20] and to two-phase flow [19,25], with suitable reduced models to describe the flow in the fracture.

However, the aforementioned works consider just the restricted case of non-intersecting fractures that cut the domain into two separated sub-domains. In [7] this assumption is relaxed to include fractures that do not cut entirely the domain, i.e. fractures with tips immersed in the enclosing porous medium, with the constraint of mesh conformity between fractures and porous medium.

Realistic simulations in a three dimensional domain are presented in [6], where suitable coupling conditions are imposed at the intersections between fractures. In particular, the continuity of pressure and mass conservation are enforced. These conditions however, also used in [5], may lead to inaccurate results if two intersecting fractures have different permeability. In this case one may expect strong variations of pressure near the intersection, thus pressure continuity does not seems an appropriate condition to represent this behavior in a model reduction approach. We mention also the recent works $[11,12]$ on three dimensional discrete fracture network flows.

In this work, we focus on the development of a reduced model that generalizes the coupling conditions of $[5,6]$ to account for different properties of the fractures such as different permeability and thickness and to include the effect of the intersection angle. The new coupling conditions allow for pressure and velocity jumps at the intersection, similarly to the conditions derived in [27] for the matrix-fracture system. Hence, we account for the fact that in a fracture system one fracture can act as a barrier or a preferential path with respect to the other. We analise the resulting coupled system of equations to derive its well posedness, and assess its conservation and positivity properties. The analysis is focused on the two dimensional case, where fractures are modeled as one dimensional manifolds. Although fractures are clearly 3D features, the study of 2D networks is still significant, particularly when the normal to the fracture mid-surface lays on a plane [23,30]. Moreover they are less computationally expensive than a three dimensional counterpart and thus useful for preliminary studies.

We propose a discretization method that allows for non matching grids at the intersection points with the intent of providing the maximal flexibility when dealing with complex networks. More precisely, we employ an extended finite element (XFEM) strategy to treat intersecting fractures.

We focus just on the fracture network neglecting flow in the surrounding medium. This choice can be regarded as an intermediate step for the development of a fully coupled model with intersecting fractures immersed in a permeable medium, but also as a reasonable approximation of realistic situations where the rock has low permeability and flow occurs mainly through the fracture network.

The paper is structured as follows. In Section 2 we introduce the governing equations and provide the setting for the derivation of the reduced model. In Section 3 the reduced model for the intersecting fractures is derived. The corresponding weak formulation in mixed form and these analyzes are presented in Section 4, 


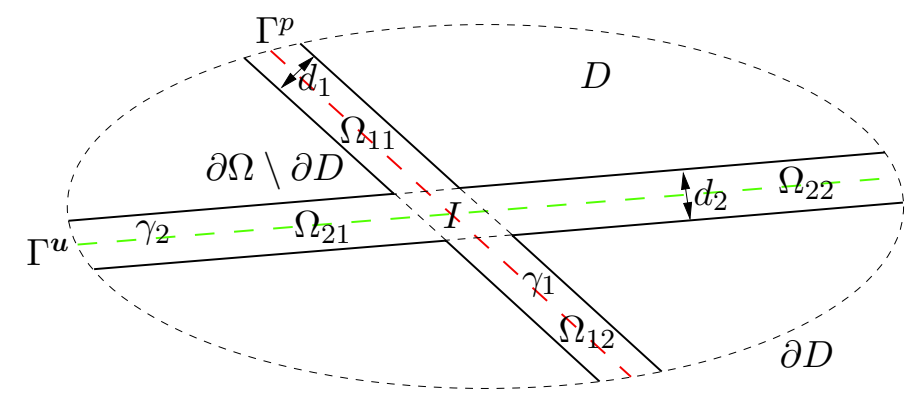

FiguRE 1. Example of a network of fractures and its subdivision (2.2).

while in Section 5 we address the numerical discretization and its main properties. In Section 6 we present some numerical test cases to assess the theoretical results. Finally, Section 7 is devoted to conclusions.

\section{The GOVERNING EQUATIONS}

In this work we consider the case of fractures in a domain of $\mathbb{R}^{2}$. For the sake of simplicity, let us consider two crossing fractures $\Omega_{1}, \Omega_{2} \in \mathbb{R}^{2}$, included in a region $D \subset \mathbb{R}^{2}$ intersecting the fracture boundary. The results illustrated in this section may be extended rather easily to the case of several fractures, as the examples in Section 6 show.

Following [27] we suppose that, for each $\Omega_{i}$, there exists a non auto-intersecting one dimensional manifold $\gamma_{i}$ of class $C^{2}$ such that $\Omega_{i}$ may be defined as

$$
\Omega_{i}=\left\{\boldsymbol{x} \in \mathbb{R}^{n}: \boldsymbol{x}=\boldsymbol{s}+r \boldsymbol{n}_{i}, \boldsymbol{s} \in \gamma_{i}, r \in\left(-\frac{d_{i}(\boldsymbol{s})}{2}, \frac{d_{i}(\boldsymbol{s})}{2}\right)\right\},
$$

where $d_{i} \in C^{2}\left(\gamma_{i}\right)$ is the thickness of $\Omega_{i}$ and $\boldsymbol{n}_{i}$ the unit normal of $\gamma_{i}$. We assume that $\left|\gamma_{i}\right| \gg d_{i}$, for $i=1,2$. Furthermore, we assume that there exist $c_{1}, c_{2} \in \mathbb{R}^{+}$, with $c_{2}$ "small", such that

$$
d_{i}(s)>c_{1}, \quad\left|d_{i}^{\prime}(s)\right|<c_{2} \quad \forall s \in \gamma_{i} \quad \text { for } i=1,2 .
$$

In other words, we assume that the thickness of the fracture varies slowly and is small compared to the other dimensions of the fracture.

Remark 2.1. The requirement that $\gamma_{i}$ be of class $C^{2}$ may be partially dispensed with. Indeed, it is sufficient that $\gamma_{i}$ be a piecewise $C^{2}$ curve.

We set $I:=\Omega_{1} \cap \Omega_{2}$ and we assume that each $\Omega_{i}$ can be subdivided into three disjoint and non-empty subregions $\Omega_{i 1}, \Omega_{i 2}$ and $I$, i.e. a $\mathrm{T}$ shaped intersection is not allowed (see Fig. 1). For convenience let us introduce the following sets, for $i, j=1,2$

$$
\bar{\Omega}:=\bar{\Omega}_{1} \cup \bar{\Omega}_{2}, \quad \bar{\gamma}:=\bar{\gamma}_{1} \cup \bar{\gamma}_{2}, \quad \boldsymbol{i}_{p}:=\gamma_{1} \cap \gamma_{2} \quad \text { and } \quad \partial I_{i j}:=\partial I \cap \partial \Omega_{i j} .
$$

It is implicit in these definitions that we assume that $\gamma_{1}$ and $\gamma_{2}$ intersect each other at a single point, indicated with $\boldsymbol{i}_{p}$. The extension to multiple intersection is straightforward.

We assume a Lipschitz-continuous boundary for both $D$ and $\Omega$. We indicate with $\boldsymbol{n}_{i j}, \boldsymbol{n}_{\Omega}$ and $\boldsymbol{n}_{D}$ the outward unit normals to $\partial I_{i j}, \partial \Omega$ and $\partial D$, respectively. Here and in the sequel we indicate with the lower case subscripts $i$ and $i j$ the restriction of data and unknowns to $\Omega_{i}$ or $\Omega_{i j}$, respectively, and with the subscript $I$ the restriction to $I$. For instance, for $\boldsymbol{u}_{i}$ in $\Omega_{i}, \boldsymbol{u}_{i j}$ indicates the function in $\Omega_{i j}$ such that $\boldsymbol{u}_{i j}=\left.\boldsymbol{u}_{i}\right|_{\Omega_{i j}}$ and so on. 
We are interested in computing the steady pressure field $p$ and the velocity field $\boldsymbol{u}$ in the whole network $\Omega$, governed by the following Darcy problems formulated in $\Omega_{i}$ and $I$,

$$
\left\{\begin{array} { l } 
{ \nabla \cdot \boldsymbol { u } _ { i } = f _ { i } } \\
{ \boldsymbol { K } _ { i } ^ { - 1 } \boldsymbol { u } _ { i } + \nabla p _ { i } = \mathbf { 0 } }
\end{array} \quad \text { in } \Omega _ { i } \backslash I \text { for } i = 1 , 2 \quad \text { and } \quad \left\{\begin{array}{ll}
\nabla \cdot \boldsymbol{u}_{I}=f_{I} \\
\boldsymbol{K}_{I}^{-1} \boldsymbol{u}_{I}+\nabla p_{I}=\mathbf{0}
\end{array} \quad \text { in } I .\right.\right.
$$

Here $\boldsymbol{K}_{i} \in\left[L^{\infty}(\Omega)\right]^{2 \times 2}$ and $\boldsymbol{K}_{I} \in\left[L^{\infty}(\Omega)\right]^{2 \times 2}$ denote the permeability tensors, which are symmetric and positive definite, and $f \in L^{2}(\Omega)$ is a source term which represents a possible volume source.

We consider the following physical coupling conditions between $I$ and $\Omega \backslash I$

$$
\left\{\begin{array}{l}
p_{i j}=p_{I} \\
\boldsymbol{u}_{i j} \cdot \boldsymbol{n}_{i j}=\boldsymbol{u}_{I} \cdot \boldsymbol{n}_{i j}
\end{array} \quad \text { on } \partial I_{i j} \text { for } i, j=1,2,\right.
$$

and boundary conditions

$$
\begin{cases}\boldsymbol{u} \cdot \boldsymbol{n}_{\Omega}=0 & \text { on } \partial \Omega \backslash \partial D \\ p=g & \text { on } \Gamma^{p} \\ \boldsymbol{u} \cdot \boldsymbol{n}_{D}=b & \text { on } \Gamma^{\boldsymbol{u}}\end{cases}
$$

The first condition of (2.3c) means that we are considering the fractures as immersed in an impermeable medium $D \backslash \Omega$. In the remaining part of $\partial \Omega$ we impose boundary condition for the pressure on $\Gamma^{p}$ with $g \in H^{1 / 2}\left(\Gamma^{p}\right)$, or for the flux on $\Gamma^{\boldsymbol{u}}$ with $b \in H^{-1 / 2}\left(\Gamma^{\boldsymbol{u}}\right)$. We have $\Gamma^{p} \cup \Gamma^{\boldsymbol{u}}:=\partial D \cap \partial \Omega$ with $\Gamma^{p} \cap \Gamma^{\boldsymbol{u}}=\emptyset$, moreover we require that $\Gamma^{p} \neq \emptyset$ and that $\partial \Omega_{i j} \cap \partial D$ belongs to $\Gamma^{p}$ or to $\Gamma^{u}$ for $i, j=1,2$. We consider a subdivision of $\Gamma^{p}$ and $\Gamma^{u}$ for each fracture, indicated as $\Gamma_{i}^{p}$ and $\Gamma_{i}^{u}$, respectively. Finally, according to the latter sub-division, we set $\partial \gamma_{i}^{p}:=\partial \gamma_{i} \cap \Gamma_{i}^{p}$ and $\partial \gamma_{i}^{u}:=\partial \gamma_{i} \cap \Gamma_{i}^{u}$. Introducing the vector functional space

$$
\mathcal{H}_{\mathrm{div}}(\Omega):=\left\{\boldsymbol{v} \in \boldsymbol{H}_{\mathrm{div}}(\Omega):\left\langle\boldsymbol{v} \cdot \boldsymbol{n}_{D}-b, w\right\rangle=0, \forall w \in H_{0, \Gamma^{u}}^{1}(\Omega)\right\},
$$

with $H_{0, \Gamma^{u}}^{1}(\Omega):=\left\{w \in H^{1}(\Omega): w=0\right.$ in $\left.\Gamma^{u}\right\}$, we have the following standard result for the Darcy problem, see $[14,16,29]$.

Theorem 2.2. Under the given hypothesis on the data, problem (2.3) is well posed. In particular, we have $(\boldsymbol{u}, p) \in \mathcal{H}_{\mathrm{div}}(\Omega) \times L^{2}(\Omega)$.

\section{Derivation of A REDUCED MODEL}

The derivation of the reduced model follows the approach presented, in a different framework, in [27].

We indicate the projection operators in the normal and tangential direction of $\gamma_{i}$ as $\boldsymbol{N}_{i}:=\boldsymbol{n}_{i} \otimes \boldsymbol{n}_{i}$ and $\boldsymbol{T}_{i}:=\boldsymbol{I}-\boldsymbol{N}_{i}$ respectively, $\boldsymbol{I}$ being the identity tensor. Given two regular functions $g$ and $\boldsymbol{q}$, we define the tangential gradient and divergence for each $\gamma_{i}$ as

$$
\nabla_{\boldsymbol{\tau}_{i}} g:=\boldsymbol{T}_{i} \nabla g \quad \text { and } \quad \nabla_{\boldsymbol{\tau}_{i}} \cdot \boldsymbol{q}:=\boldsymbol{T}_{i}: \nabla \boldsymbol{q},
$$

respectively. We require that the permeability tensor $\boldsymbol{K}_{i}$ in $\Omega_{i} \backslash I, i=1,2$, can be written as $\boldsymbol{K}_{i}=K_{i, \boldsymbol{n}} \boldsymbol{N}_{i}+$ $K_{i, \boldsymbol{\tau}} \boldsymbol{T}_{i}$, with $K_{i, \boldsymbol{n}}, K_{i, \boldsymbol{\tau}} \in L^{\infty}\left(\Omega_{i j}\right)$ and strictly positive. This is a reasonable request since we are assuming, in the two-dimensional case, that the permeability tensor is diagonal in a frame of reference that is aligned with the fracture. In the three dimensional case this assumption also implies that the permeability should be isotropic in the tangent plane of the fracture. 


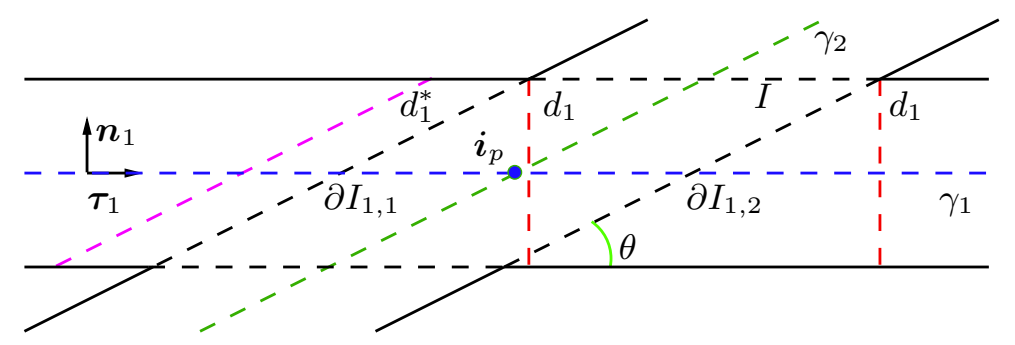

Figure 2. Example of an intersection.

We indicate with the symbol $\hat{\imath}$ the reduced variables defined on $\gamma_{i}$. In particular, for any $\boldsymbol{s}_{i} \in \gamma_{i}$, we introduce the reduced pressure $\hat{p}$ and flux $\hat{\boldsymbol{u}}$ as

$$
\hat{\boldsymbol{u}}_{i}\left(\boldsymbol{s}_{i}\right):=\int_{-\frac{d_{i}}{2}}^{\frac{d_{i}}{2}} \boldsymbol{T}_{i} \boldsymbol{u}_{i}\left(\boldsymbol{s}_{i}+r \boldsymbol{n}_{i}\right) \mathrm{d} r \quad \text { and } \quad \hat{p}_{i}\left(\boldsymbol{s}_{i}\right):=\frac{1}{d_{i}} \int_{-\frac{d_{i}}{2}}^{\frac{d_{i}}{2}} p_{i}\left(\boldsymbol{s}_{i}+r \boldsymbol{n}_{i}\right) \mathrm{d} r .
$$

Note that we have preferred to use the flux and not the average velocity in the reduced model since it simplifies the model in the case of fractures with variable thickness.

Moreover, a reduced source term and the inverse of the scaled permeabilities are defined as

$$
\hat{f}_{i}\left(\boldsymbol{s}_{i}\right):=\int_{-\frac{d_{i}}{2}}^{\frac{d_{i}}{2}} f_{i}\left(\boldsymbol{s}_{i}+r \boldsymbol{n}_{i}\right) \mathrm{d} r, \quad \eta_{\gamma_{i}}:=\frac{d_{i}}{K_{i, \boldsymbol{n}}} \quad \text { and } \quad \hat{\eta}_{i}:=\frac{1}{d_{i} K_{i, \boldsymbol{\tau}}},
$$

respectively.

Using (3.2) and approximating $\Gamma_{i}^{\boldsymbol{u}}$ with $\partial \gamma_{i}^{\boldsymbol{u}}$ and $\Gamma_{i}^{p}$ with $\partial \gamma_{i}^{p}$, so that $\partial \gamma_{i}^{\boldsymbol{u}} \cap \partial \gamma_{i}^{p}=\emptyset$, we obtain the corresponding reduced boundary data from the last two expressions of $(2.3 \mathrm{c})$,

$$
\hat{b}_{i j}:=\int_{\partial \Omega_{i j} \cap \partial D} b_{i j}(\boldsymbol{\sigma}) \mathrm{d} \boldsymbol{\sigma} \quad \text { and } \quad \hat{g}_{i j}:=\frac{1}{\left|\partial \Omega_{i j} \cap \partial D\right|} \int_{\partial \Omega_{i j} \cap \partial D} g_{i j}(\boldsymbol{\sigma}) \mathrm{d} \boldsymbol{\sigma} .
$$

Indeed, by the definition in (3.2) we have that $\hat{\boldsymbol{u}}_{i} \cdot \boldsymbol{n}_{i}=0$ on $\gamma_{i}$, i.e. $\hat{\boldsymbol{u}}_{i}$ is aligned to the tangent of $\gamma_{i}$.

The reduced model on each $\Omega_{i j}$ is obtained by integrating (2.3a) along the fracture thickness and can then be written as

$$
\left\{\begin{array} { l } 
{ \nabla _ { \boldsymbol { \tau } _ { i } } \cdot \hat { \boldsymbol { u } } _ { i } = \hat { f } _ { i } } \\
{ \hat { \eta } _ { i } \hat { \boldsymbol { u } } _ { i } + \nabla _ { \boldsymbol { \tau } _ { i } } \hat { p } _ { i } = \mathbf { 0 } }
\end{array} \quad \text { in } \gamma _ { i } \backslash \boldsymbol { i } _ { p } \quad \text { and } \quad \left\{\begin{array}{ll}
\hat{\boldsymbol{u}}_{i} \cdot \boldsymbol{n}_{D}=\hat{b}_{i} & \text { on } \partial \gamma_{i}^{\boldsymbol{u}} \\
\hat{p}_{i}=\hat{g}_{i} & \text { on } \partial \gamma_{i}^{p}
\end{array} \text { for } i=1,2 .\right.\right.
$$

We derive now a reduced model for the flow in the intersecting region $I$ in order to find proper coupling conditions. To this aim, we assume that $I$ can be modeled as a quadrilateral with parallel sides, i.e. the thicknesses $d_{i}$ can be considered constant in $I$. Furthermore, we have assumed that the permeability tensor $\boldsymbol{K}_{I}$ can be taken constant in $I$. Let us indicate with $\boldsymbol{\tau}_{i}$ the tangential unit vector to $\gamma_{i}$, and with $\boldsymbol{\tau}_{i, \boldsymbol{i}_{p}}$ its value at $\boldsymbol{i}_{p}$, and define $d_{i}^{*}:=d_{i} / \sin \theta$, with $\sin \theta=\sqrt{1-\left(\boldsymbol{\tau}_{1, \boldsymbol{i}_{p}} \cdot \boldsymbol{\tau}_{2, \boldsymbol{i}_{p}}\right)^{2}}$. Then $|I|=d_{1}^{*} d_{2}^{*}|\sin \theta|$. Note that $\theta$ is the angle between fractures at the intersection, as shown in Figure 2. We can write the intersecting region as

$$
I=\left\{\boldsymbol{x} \in \Omega: \boldsymbol{x}=\boldsymbol{i}_{p}+x_{1} \boldsymbol{\tau}_{1, \boldsymbol{i}_{p}}+x_{2} \boldsymbol{\tau}_{2, \boldsymbol{i}_{p}}, x_{i} \in\left(-\frac{d_{j}^{*}}{2}, \frac{d_{j}^{*}}{2}\right) \text { for } i \neq j=1,2\right\} .
$$


The reduction process approximates $I$ with $\boldsymbol{i}_{p}$ and assumes that the fluxes $\hat{\boldsymbol{u}}_{i}$ and the pressures $\hat{p}_{i}$ can be discontinuous at $\boldsymbol{i}_{p}$. Thus, we denote with $\hat{p}_{I} \in \mathbb{R}$ the reduced value of the pressure at the intersection, defined as

$$
\hat{p}_{I}:=\frac{1}{|I|} \int_{I} p_{I}(\boldsymbol{x}) \mathrm{d} \boldsymbol{x} .
$$

The intersection point divides each line $\gamma_{i}$ into two parts, $\gamma_{i 1}$ and $\gamma_{i 2}$ respectively, where the indexes 1 and 2 refer to the orientation induced by the tangent vectors. Again, a double subscript $i j$, with $i, j=1,2$, will be used to indicate quantities on $\gamma_{i j}$. Furthermore, since $\hat{\boldsymbol{u}}_{i}$ is by definition aligned along $\gamma_{i}$ we may write $\hat{\boldsymbol{u}}_{i}=u_{i} \boldsymbol{\tau}_{i}$.

We can then define the jump and mean operator across $\boldsymbol{i}_{p}$ as

$$
\llbracket a_{i} \rrbracket_{\boldsymbol{i}_{p}}:=a_{i 1}-a_{i 2} \quad \text { and } \quad\left\{\left\{a_{i}\right\}_{\boldsymbol{i}_{p}}:=\frac{a_{i 1}+a_{i 2}}{2}, \quad \text { for } \quad i=1,2 .\right.
$$

It is reasonable to make the following approximation, $(2.3 \mathrm{~b})$

$$
\left(\int_{\partial I_{i j}} \boldsymbol{u}_{I} \cdot \mathbf{n}\right) \boldsymbol{\tau}_{i} \approx \hat{\boldsymbol{u}}_{i j}\left(\boldsymbol{i}_{p}\right) \quad \text { and } \quad \frac{1}{d_{i}^{*}} \int_{\partial I_{i j}} p_{I} \approx \hat{p}_{i j}\left(\boldsymbol{i}_{p}\right), \quad \text { for } i, j=1,2 .
$$

Mass conservation implies that

$$
\sum_{k=1}^{2} \llbracket \hat{\boldsymbol{u}}_{k} \cdot \boldsymbol{\tau}_{k} \rrbracket_{\boldsymbol{i}_{p}}=\hat{f}_{I} \quad \text { with } \quad \hat{f}_{I}:=\int_{I} f_{I}(\boldsymbol{x}) \mathrm{d} \boldsymbol{x} .
$$

We integrate the first of (2.3a) on $I$, approximating the integral involving the velocity $\boldsymbol{u}_{I}$ by the trapezoidal rule on each fracture, to find

$$
\int_{I} \boldsymbol{K}_{I}^{-1} \boldsymbol{u}_{I} \approx \boldsymbol{K}_{I}^{-1} \frac{|I|}{2} \sum_{k=1}^{2} \frac{1}{d_{k}^{*}}\left(\hat{\boldsymbol{u}}_{k 1}+\hat{\boldsymbol{u}}_{k 2}\right)=\boldsymbol{K}_{I}^{-1}|I| \sum_{k=1}^{2} \frac{1}{d_{k}^{*}}\left\{\left\{\hat{\boldsymbol{u}}_{k}\right\}_{\boldsymbol{i}_{p}} .\right.
$$

Furthermore, the integral of the gradient of the pressure $p_{I}$ in the intersection can be written as

$$
\int_{I} \nabla p_{I}=\sum_{i, j=1}^{2} \boldsymbol{n}_{i j} \int_{\partial I_{i j}} p_{I} \approx\left(\hat{p}_{12}-\hat{p}_{11}\right) \boldsymbol{n}_{2} d_{1}^{*}+\left(\hat{p}_{22}-\hat{p}_{21}\right) \boldsymbol{n}_{1} d_{2}^{*}=-\llbracket \hat{p}_{1} \rrbracket_{\boldsymbol{i}_{p}} \boldsymbol{n}_{2} d_{1}^{*}-\llbracket \hat{p}_{2} \rrbracket_{\boldsymbol{i}_{p}} \boldsymbol{n}_{1} d_{2}^{*}
$$

Then, we obtain

$$
\boldsymbol{K}_{I}^{-1}|I| \sum_{k=1}^{2} \frac{1}{d_{k}^{*}}\left\{\left\{\hat{\boldsymbol{u}}_{k} \rrbracket_{\boldsymbol{i}_{p}}=\llbracket \hat{p}_{1} \rrbracket_{\boldsymbol{i}_{p}} \boldsymbol{n}_{2} d_{1}^{*}+\llbracket \hat{p}_{2} \rrbracket_{\boldsymbol{i}_{p}} \boldsymbol{n}_{1} d_{2}^{*} .\right.\right.
$$

Multiplying the above relation by $\boldsymbol{\tau}_{1}$, or similarly by $\boldsymbol{\tau}_{2}$, using the identity $d_{1}=d_{1}^{*} \boldsymbol{n}_{2} \cdot \boldsymbol{\tau}_{1}$ and the fact that $\hat{\boldsymbol{u}}_{i}=\left(\hat{\boldsymbol{u}}_{i} \cdot \boldsymbol{\tau}_{i}\right) \boldsymbol{\tau}_{i}$ we obtain,

$$
\frac{|I|}{d_{i}} \sum_{k=1}^{2} \frac{\eta_{i k}^{I}}{d_{k}^{*}}\left\{\left\{\hat{\boldsymbol{u}}_{k} \cdot \boldsymbol{\tau}_{k}\right\}_{\boldsymbol{i}_{p}}=\llbracket \hat{p}_{i} \rrbracket_{\boldsymbol{i}_{p}} \quad \text { in } \boldsymbol{i}_{p}, \quad i=1,2,\right.
$$

where

$$
\eta_{i j}^{I}:=\boldsymbol{\tau}_{i, i_{p}}^{\top} \cdot \boldsymbol{K}_{I}^{-1} \boldsymbol{\tau}_{j, i_{p}}
$$




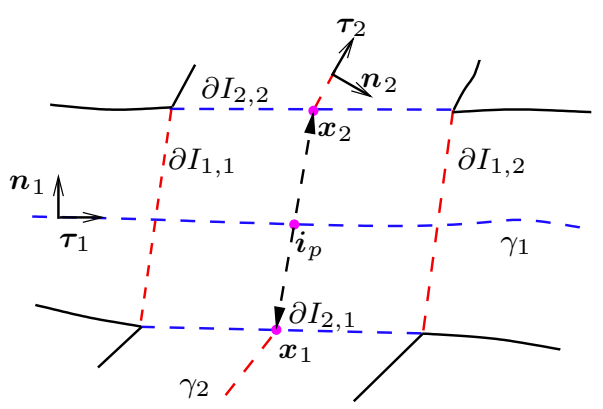

FiguRE 3. Example of a bi-dimensional intersection between two fractures.

If $\gamma_{1}$ and $\gamma_{2}$ are orthogonal and the permeability tensor $\boldsymbol{K}_{I}$ is such that

$$
\boldsymbol{K}_{I}^{-1}=\eta_{\gamma_{1}} \boldsymbol{\tau}_{1, i_{p}} \otimes \boldsymbol{\tau}_{1, i_{p}}+\eta_{\gamma_{2}} \boldsymbol{\tau}_{2, i_{p}} \otimes \boldsymbol{\tau}_{2, i_{p}}
$$

coupling conditions (3.4) can be simplified into

$$
\eta_{\gamma_{i}} \frac{d_{j}}{d_{i}}\left\{\left\{\hat{\boldsymbol{u}}_{i} \cdot \boldsymbol{\tau}_{i}\right\}_{\boldsymbol{i}_{p}}=\llbracket \hat{p}_{i} \rrbracket_{\boldsymbol{i}_{p}} \quad \text { in } \boldsymbol{i}_{p}, \text { for } i, j=1,2, \quad j \neq i .\right.
$$

To close the system we derive a model for the pressure at the intersection. For each fracture in the first half of the transverse section we approximate the value of the pressure in $\boldsymbol{i}_{p}$ by the following truncated Taylor expansion,

$$
p_{j}\left(\boldsymbol{i}_{p}\right)=p_{I}\left(\boldsymbol{x}_{1}\right)+\frac{d_{j}^{*}}{2} \nabla p_{I}\left(\boldsymbol{\theta}_{1}\right) \cdot \boldsymbol{\tau}_{i} \quad i, j=1,2 \quad \text { with } i \neq j,
$$

where $\boldsymbol{\theta}_{1}=\boldsymbol{i}_{p}-\boldsymbol{\tau}_{i} \xi_{1} d_{j}^{*} / 2$ with $\xi_{1} \in[0,1]$, see Figure 3 . In the second transverse section we use an analogous approximation, namely

$$
p_{j}\left(\boldsymbol{i}_{p}\right)=p_{I}\left(\boldsymbol{x}_{2}\right)-\frac{d_{j}^{*}}{2} \nabla p_{I}\left(\boldsymbol{\theta}_{2}\right) \cdot \boldsymbol{\tau}_{i} \quad i, j=1,2 \quad \text { with } i \neq j,
$$

where $\boldsymbol{\theta}_{2}=\boldsymbol{i}_{p}+\boldsymbol{\tau}_{i} \xi_{2} d_{j}^{*} / 2$ with $\xi_{2} \in[0,1]$. Using (2.3a) and (2.3b) we find

$$
p_{j}\left(\boldsymbol{i}_{p}\right)=p_{i, k}+(-1)^{k} \frac{d_{j}^{*}}{2} \boldsymbol{\tau}_{i}^{\top} \cdot \boldsymbol{K}_{I}^{-1} \boldsymbol{u}_{I}\left(\boldsymbol{\theta}_{k}\right) \quad \text { for } k=1,2 .
$$

The values of $\boldsymbol{u}_{I}$ in both $\boldsymbol{\theta}_{1}$ and $\boldsymbol{\theta}_{2}$ are unknown, therefore we express them by the following convex combination for each fracture

$$
\begin{array}{ll}
\boldsymbol{u}_{I}\left(\boldsymbol{\theta}_{1}\right)=\xi_{1} \boldsymbol{u}_{1,1}+\left(1-\xi_{1}\right) \boldsymbol{u}_{1,2}+\frac{1}{2}\left(\boldsymbol{u}_{2,1}+\boldsymbol{u}_{2,2}\right) & \text { for } \xi_{1} \in[0,1], \\
\boldsymbol{u}_{I}\left(\boldsymbol{\theta}_{2}\right)=\xi_{2} \boldsymbol{u}_{1,2}+\left(1-\xi_{2}\right) \boldsymbol{u}_{1,1}+\frac{1}{2}\left(\boldsymbol{u}_{2,2}+\boldsymbol{u}_{2,1}\right) & \text { for } \xi_{2} \in[0,1]
\end{array}
$$

Using the previous expression for $\boldsymbol{u}_{I}$ and integrating in $I$, equation (3.9) becomes

$$
\begin{aligned}
& \hat{p}_{I}=\hat{p}_{i, 1}-\boldsymbol{\tau}_{i}^{\top} \cdot \boldsymbol{K}_{I}^{-1}\left[\frac { 1 } { 2 } \left(\frac{d_{j}}{d_{i}}\left\{\left\{\hat{\boldsymbol{u}}_{i}\right\}_{\boldsymbol{i}_{p}}+\left\{\left\{\hat{\boldsymbol{u}}_{j}\right\}_{\boldsymbol{i}_{p}}\right)+\hat{\xi}_{0,1} \frac{d_{j}}{d_{i}} \llbracket \hat{\boldsymbol{u}}_{i} \rrbracket_{\boldsymbol{i}_{p}}\right],\right.\right. \\
& \hat{p}_{I}=\hat{p}_{i, 2}+\boldsymbol{\tau}_{i}^{\top} \cdot \boldsymbol{K}_{I}^{-1}\left[\frac { 1 } { 2 } \left(\frac{d_{j}}{d_{i}}\left\{\left\{\hat{\boldsymbol{u}}_{i}\right\}_{\boldsymbol{i}_{p}}+\left\{\left\{\hat{\boldsymbol{u}}_{j}\right\}_{\boldsymbol{i}_{p}}\right)-\hat{\xi}_{0,2} \frac{d_{j}}{d_{i}} \llbracket \hat{\boldsymbol{u}}_{i} \rrbracket_{\boldsymbol{i}_{p}}\right],\right.\right.
\end{aligned}
$$


where $\hat{\xi}_{0, k}:=\left(2 \xi_{k}-1\right) / 4$ for $k=1,2$. Finally using (3.4) and the fact that pressure $\hat{p}_{I}$ is single valued, thus $\hat{\xi}_{0, k}=\hat{\xi}_{0}$ for $k=1,2$, we obtain the last coupling condition of our reduced model

$$
\hat{\xi}_{0} \frac{d_{j}}{d_{i}} \eta_{i i}^{I} \llbracket \hat{\boldsymbol{u}}_{i} \cdot \boldsymbol{\tau}_{i} \rrbracket_{\boldsymbol{i}_{p}}=\left\{\left\{\hat{p}_{i}\right\}_{\boldsymbol{i}_{p}}-\hat{p}_{I} \quad \text { in } \boldsymbol{i}_{p} .\right.
$$

To sum up, the complete reduced model that describes the evolution of $\hat{\boldsymbol{u}}_{i}, \hat{p}_{i}$ and $\hat{p}_{I}$ consists of the following system of partial differential equations

$$
\left\{\begin{array} { l } 
{ \nabla _ { \boldsymbol { \tau } _ { i } } \cdot \hat { \boldsymbol { u } } _ { i } = \hat { f } _ { i } } \\
{ \hat { \eta } _ { i } \hat { \boldsymbol { u } } _ { i } + \nabla _ { \boldsymbol { \tau } _ { i } } \hat { p } _ { i } = \mathbf { 0 } }
\end{array} \quad \text { in } \gamma _ { i } \backslash \boldsymbol { i } _ { p } \quad \text { and } \quad \left\{\begin{array}{ll}
\hat{\boldsymbol{u}}_{i} \cdot \boldsymbol{n}_{D}=\hat{b}_{i} & \text { on } \partial \gamma_{i}^{\boldsymbol{u}} \\
\hat{p}_{i}=\hat{g}_{i} & \text { on } \partial \gamma_{i}^{p}
\end{array} \text { for } i=1,2 .\right.\right.
$$

and the coupling conditions for the fracture-fracture system

$$
\left\{\begin{array}{l}
\sum_{k=1}^{2} \llbracket \hat{\boldsymbol{u}}_{k} \cdot \boldsymbol{\tau}_{k} \rrbracket_{\boldsymbol{i}_{p}}=\hat{f}_{I} \\
\frac{|I|}{d_{i}} \sum_{k=1}^{2} \frac{\eta_{i k}^{I}}{d_{k}^{*}}\left\{\left\{\hat{\boldsymbol{u}}_{k} \cdot \boldsymbol{\tau}_{k}\right\}_{\boldsymbol{i}_{p}}=\llbracket \hat{p}_{i} \rrbracket_{\boldsymbol{i}_{p}} \quad \text { in } \boldsymbol{i}_{p}, i, j=1,2, i \neq j .\right. \\
\hat{\xi}_{0} \frac{d_{j}}{d_{i}} \eta_{i i}^{I} \llbracket \hat{\boldsymbol{u}}_{i} \cdot \boldsymbol{\tau}_{i} \rrbracket_{\boldsymbol{i}_{p}}=\left\{\hat{p}_{i}\right\}_{\boldsymbol{i}_{p}}-\hat{p}_{I}
\end{array}\right.
$$

If the intersection region has a high permeability then $\eta_{i j}^{I} \approx 0$ and conditions (3.11b) reduce to those in $[4,6]$, i.e. continuity of pressure and mass conservation. However, our model is more general as it allows for different choices of $\boldsymbol{K}_{I}$, and it is useful in practical situations where fractures have rather different permeability and may even act as barrier to the flow. This fact will be illustrated in the section dedicated to numerical experimentation.

\section{WEAK FORMULATION AND FUNCTIONAL SETTING}

We describe here the functional setting for homogeneous essential boundary conditions, i.e. all possible $\hat{b}_{i}$ are set to zero, since the non-homogeneous case may be recovered by standard lifting techniques. For a given regular curve $\gamma:(0, L) \rightarrow \mathbb{R}^{2}$ with tangent $\boldsymbol{\tau}$ defined almost everywhere on $\gamma$ we define the vector space

$$
\boldsymbol{H}_{\mathrm{div}}(\gamma):=\left\{\boldsymbol{w} \in\left[L^{2}(\gamma)\right]^{2}: \nabla_{\boldsymbol{\tau}} \cdot \boldsymbol{w} \in L^{2}(\gamma), \boldsymbol{w} \cdot \boldsymbol{n}=0 \text { and } \boldsymbol{w} \cdot \boldsymbol{n}_{D}=0 \text { on } \partial \gamma\right\},
$$

with norm

$$
\|\boldsymbol{w}\|_{\boldsymbol{H}_{\text {div }}(\gamma)}^{2}:=\|\boldsymbol{w}\|_{L^{2}(\gamma)}^{2}+\left\|\nabla_{\boldsymbol{\tau}} \cdot \boldsymbol{w}\right\|_{L^{2}(\gamma)}^{2} .
$$

Furthermore, we assume that elements of $\boldsymbol{w} \in \boldsymbol{H}_{\operatorname{div}}(\gamma)$ are aligned with $\gamma$, i.e. for a $\boldsymbol{w} \in \boldsymbol{H}_{\operatorname{div}}(\gamma)$ we have $\boldsymbol{w}=w \boldsymbol{\tau}$ with $w \in H^{1}(\gamma)$. We set $\boldsymbol{W}_{i j}:=\boldsymbol{H}_{\mathrm{div}}\left(\gamma_{i j}\right)$ and $\boldsymbol{W}_{i}:=\boldsymbol{W}_{i 1} \times \boldsymbol{W}_{i 2}$ with norm

$$
\left\|\boldsymbol{w}_{i}\right\|_{\boldsymbol{W}_{i}}^{2}:=\left\|\boldsymbol{w}_{i 1}\right\|_{\boldsymbol{W}_{i 1}}^{2}+\left\|\boldsymbol{w}_{i 2}\right\|_{\boldsymbol{W}_{i 2}}^{2}+\llbracket \boldsymbol{w}_{i} \cdot \boldsymbol{\tau}_{i} \rrbracket_{\boldsymbol{i}_{p}}^{2} \quad \text { where } \quad \boldsymbol{w}_{i}=\left(\boldsymbol{w}_{i 1}, \boldsymbol{w}_{i 2}\right) \in \boldsymbol{W}_{i} .
$$

Let us define $Q_{i j}:=L^{2}\left(\gamma_{i j}\right)$ and $Q_{i}:=Q_{i 1} \times Q_{i 2}$. $Q_{i}$ may be identified with $L^{2}\left(\gamma_{i}\right)$. We set $\boldsymbol{W}:=\boldsymbol{W}_{1} \times \boldsymbol{W}_{2}$ with norm

$$
\|\boldsymbol{w}\|_{\boldsymbol{W}}^{2}:=\left\|\boldsymbol{w}_{1}\right\|_{\boldsymbol{W}_{1}}^{2}+\left\|\boldsymbol{w}_{2}\right\|_{\boldsymbol{W}_{2}}^{2}, \quad \text { where } \quad \boldsymbol{w}=\left(\boldsymbol{w}_{1}, \boldsymbol{w}_{2}\right) \in \boldsymbol{W},
$$

and $Q:=Q_{1} \times Q_{2} \times \mathbb{R}$ with norm

$$
\|q\|_{Q}^{2}:=\left\|q_{1}\right\|_{Q_{1}}^{2}+\left\|q_{2}\right\|_{Q_{2}}^{2}+q_{3}^{2}, \quad \text { where } \quad q=\left(q_{1}, q_{2}, q_{3}\right) \in Q .
$$

All those spaces are in fact Hilbert spaces equipped with scalar products associated with the chosen norms. 
To obtain the weak formulation of (3.11) we take a test function $q_{i} \in Q_{i}$ and integrate on each branch $\gamma_{i j}$ the first equation in (3.11a) to obtain, summing over $j$

$$
\left(\nabla_{\tau_{i}} \cdot \hat{\boldsymbol{u}}_{i}, q_{i}\right)_{\gamma_{i}}=\left(\hat{f}_{i}, q_{i}\right)_{\gamma_{i}} \forall q_{i} \in Q_{i}, i=1,2
$$

Taking then a test function $\boldsymbol{w}_{i} \in \boldsymbol{W}_{i}$ and integrating on each $\gamma_{i j}$ the second equation in (3.11a), we obtain

$$
\left(\hat{\eta}_{i} \hat{\boldsymbol{u}}_{i}, \boldsymbol{w}_{i}\right)_{\gamma_{i}}-\left(\hat{p}_{i}, \nabla_{\tau_{i}} \cdot \boldsymbol{w}_{i}\right)_{\gamma_{i}}+\llbracket \hat{p}_{i} \boldsymbol{w}_{i} \cdot \boldsymbol{\tau}_{i} \rrbracket_{i_{p}}+\left.\sum_{i j: \partial \gamma_{i j}^{p} \neq \emptyset} \hat{g}_{i}\left(\boldsymbol{w}_{i} \cdot \boldsymbol{n}_{D}\right)\right|_{\partial \gamma_{i j}^{p}}=0 \quad \forall \boldsymbol{w}_{i} \in \boldsymbol{W}_{i}, i=1,2 .
$$

Note that we have integrated by parts the pressure term and used the natural boundary conditions. Thanks to the identity $\llbracket a b \rrbracket=\llbracket a \rrbracket\{b b\}+\{\{a \rrbracket \llbracket b \rrbracket$ we can include the coupling conditions (3.11b) substituting the expression of the pressure jump and average as

$$
\llbracket \hat{p}_{i} \boldsymbol{w}_{i} \cdot \boldsymbol{\tau}_{i} \rrbracket_{\boldsymbol{i}_{p}}=\llbracket \hat{p}_{i} \rrbracket_{\boldsymbol{i}_{p}}\left\{\left\{\boldsymbol{w}_{i} \cdot \boldsymbol{\tau}_{i}\right\}_{\boldsymbol{i}_{p}}+\left\{\left\{\hat{p}_{i}\right\}_{\boldsymbol{i}_{p}} \llbracket \boldsymbol{w}_{i} \cdot \boldsymbol{\tau}_{i} \rrbracket_{\boldsymbol{i}_{p}} .\right.\right.
$$

Introducing $\hat{\boldsymbol{u}}:=\left(\hat{\boldsymbol{u}}_{1}, \hat{\boldsymbol{u}}_{2}\right) \in \boldsymbol{W}$ and $\hat{p}:=\left(\hat{p}_{1}, \hat{p}_{2}, \hat{p}_{I}\right) \in Q$ and summing over $i$, the weak formulation of the coupled problem (3.11) can be now written as: find $(\hat{\boldsymbol{u}}, \hat{p}) \in \boldsymbol{W} \times Q$ such that

$$
\begin{cases}\mathcal{A}(\hat{\boldsymbol{u}}, \boldsymbol{w})+\mathcal{B}(\hat{p}, \boldsymbol{w})=\mathcal{F}(\boldsymbol{w}) & \forall \boldsymbol{w} \in \boldsymbol{W} \\ \mathcal{B}(q, \hat{\boldsymbol{u}})=\mathcal{Q}(q) & \forall q \in Q\end{cases}
$$

The functionals and bilinear forms in (4.1) are defined as

$$
\begin{aligned}
\mathcal{A}(\boldsymbol{u}, \boldsymbol{w}) & :=\sum_{i=1}^{2} a_{i}\left(\boldsymbol{u}_{i}, \boldsymbol{w}_{i}\right)+\sum_{\substack{i, j=1 \\
i \neq j}}^{2} \eta_{i j}^{I}\left\{\{ \boldsymbol { u } _ { j } \cdot \boldsymbol { \tau } _ { j } \} _ { \boldsymbol { i } _ { p } } \left\{\left\{\boldsymbol{w}_{j} \cdot \boldsymbol{\tau}_{j}\right\}_{\boldsymbol{i}_{p}},\right.\right. \\
\mathcal{B}(q, \boldsymbol{w}) & :=\sum_{i=1}^{2}-\left(q_{i}, \nabla_{\boldsymbol{\tau}_{i}} \cdot \boldsymbol{w}_{i}\right)_{L^{2}\left(\gamma_{i}\right)}+q_{3} \llbracket \boldsymbol{w}_{i} \cdot \boldsymbol{\tau}_{i} \rrbracket_{\boldsymbol{i}_{p}}, \\
\mathcal{F}(\boldsymbol{w}) & :=\sum_{i j: \partial \gamma_{i j}^{p} \neq \emptyset}-\left.\hat{g}_{i}\left(\boldsymbol{w}_{i} \cdot \boldsymbol{n}_{D}\right)\right|_{\partial \gamma_{i j}^{p}} \\
\mathcal{Q}(q) & :=\sum_{i=1}^{2}-\left(\hat{f}_{i}, q_{i}\right)_{L^{2}\left(\gamma_{i}\right)}+\hat{f}_{I} q_{3} .
\end{aligned}
$$

The bilinear forms $a_{i}$ in $\mathcal{A}$ are given, for $i, j=1,2$ and $i \neq j$, by

$$
a_{i}(\boldsymbol{u}, \boldsymbol{w}):=\left(\hat{\eta}_{i} \boldsymbol{u}, \boldsymbol{w}\right)_{L^{2}\left(\gamma_{i}\right)}+\eta_{i i}^{I} \frac{d_{j}}{d_{i}}\left(\hat{\xi}_{0} \llbracket \boldsymbol{u} \cdot \boldsymbol{\tau}_{i} \rrbracket_{\boldsymbol{i}_{p}} \llbracket \boldsymbol{w} \cdot \boldsymbol{\tau}_{i} \rrbracket_{\boldsymbol{i}_{p}}+\left\{\left\{\boldsymbol{u} \cdot \boldsymbol{\tau}_{i}\right\}_{\boldsymbol{i}_{p}}\left\{\left\{\boldsymbol{w} \cdot \boldsymbol{\tau}_{i}\right\}_{\boldsymbol{i}_{p}}\right) .\right.\right.
$$

We have the following

Lemma 4.1 (Boundedness of $\mathcal{A}$ ). There exist a constant $C \in \mathbb{R}^{+}$such that

$$
|\mathcal{A}(\boldsymbol{u}, \boldsymbol{w})| \leq C\|\boldsymbol{u}\|_{W}\|\boldsymbol{w}\|_{W} \quad \forall \boldsymbol{u}, \boldsymbol{w} \in \boldsymbol{W} .
$$

Proof. $\mathcal{A}$ is clearly a bilinear form on $\boldsymbol{W}$. Since each $\boldsymbol{u}_{i j}$ and $\boldsymbol{w}_{i j}$ are aligned along $\gamma_{i j}$, that is $\boldsymbol{u}_{i j}=u_{i j} \boldsymbol{\tau}_{i}$, the request that $\boldsymbol{u}_{i j} \in \boldsymbol{H}_{\text {div }}\left(\gamma_{i j}\right)$ implies that $u_{i j} \in H^{1}\left(\gamma_{i j}\right)$. Then the boundedness of $\mathcal{A}$ can be obtained from 
the application Cauchy-Schwarz inequality together with Sobolev embeddings and trace inequalities [1,13], by which we can finally state that $\exists C \in \mathbb{R}^{+}$such that

$$
|\mathcal{A}(\boldsymbol{u}, \boldsymbol{w})| \leq C\|\boldsymbol{u}\|_{\boldsymbol{W}}\|\boldsymbol{w}\|_{\boldsymbol{W}} \quad \text { where } \quad C=C\left(\|\hat{\eta}\|_{L^{\infty}\left(\gamma_{1} \cup \gamma_{2}\right)}, \lambda_{\max }, \bar{d}, c_{\gamma}\right)
$$

with $\bar{d}:=\max _{i \neq j=1,2} d_{i} / d_{j}$ and $c_{\gamma}$ depends on the trace inequality constants for each $\gamma_{i j}$.

Lemma 4.2 (Coercivity of $\mathcal{A})$. There exist a constant $\alpha \in \mathbb{R}^{+}$such that

$$
\mathcal{A}(\boldsymbol{u}, \boldsymbol{u}) \geq \alpha\|\boldsymbol{u}\|_{\boldsymbol{W}}^{2} \quad \forall \boldsymbol{u} \in \boldsymbol{W}_{0}
$$

where $\boldsymbol{W}_{0}:=\{\boldsymbol{w} \in \boldsymbol{W}: \mathcal{B}(q, \boldsymbol{w})=0, \forall q \in Q\}$.

Proof. By definition of $\mathcal{B}$ we have that a $\boldsymbol{w} \in \boldsymbol{W}_{0}$ is characterized by $\nabla_{\boldsymbol{\tau}_{i}} \cdot \boldsymbol{w}_{i j}=0$ almost everywhere on $\gamma_{i j}$ and $\llbracket \boldsymbol{w}_{i} \cdot \boldsymbol{\tau}_{i} \rrbracket_{\boldsymbol{i}_{p}}=0$, for $i, j=1,2$. Therefore, for a $\boldsymbol{w} \in \boldsymbol{W}_{0}$ we have $\|\boldsymbol{w}\|_{\boldsymbol{W}}^{2}=\sum_{i=1}^{2}\left\|\boldsymbol{w}_{i}\right\|_{L^{2}\left(\gamma_{i}\right)}^{2}$. Moreover, if $\boldsymbol{w} \in \boldsymbol{W}_{0}$ we have

$$
\mathcal{A}(\boldsymbol{w}, \boldsymbol{w})=\sum_{i=1}^{2}\left\|\eta_{i}^{1 / 2} \boldsymbol{w}_{i}\right\|_{L^{2}\left(\gamma_{i}\right)}^{2}+\sum_{\substack{i, j=1 \\ i \neq j}}^{2}\left(\eta _ { i i } ^ { I } \frac { d _ { j } } { d _ { i } } \left\{\left\{\boldsymbol{w}_{i} \cdot \boldsymbol{\tau}_{i}\right\}_{\boldsymbol{i}_{p}}^{2}+\eta_{i j}^{I}\left\{\left\{\boldsymbol{w}_{i} \cdot \boldsymbol{\tau}_{i}\right\}\right\}_{\boldsymbol{i}_{p}}\left\{\left\{\boldsymbol{w}_{j} \cdot \boldsymbol{\tau}_{j}\right\}_{\boldsymbol{i}_{p}}\right) .\right.\right.
$$

Let us introduce the vectors $\boldsymbol{a}_{i}=\sqrt{d_{j} / d_{i}}\left\{\left\{\boldsymbol{w}_{i} \cdot \boldsymbol{\tau}_{i}\right\}_{\boldsymbol{i}_{p}} \boldsymbol{\tau}_{i}\right.$, for $i, j=1,2$ and $j \neq i$ and the scalar product $\left(\boldsymbol{a}_{1}, \boldsymbol{a}_{2}\right)_{K}=\boldsymbol{a}_{1}^{T} \boldsymbol{K}_{I}^{-1} \boldsymbol{a}_{2}$. We recall the definition of $\eta_{i j}^{I}$ in (3.5) to note that the last sum in the previous equality may be written as

$$
\left(\boldsymbol{a}_{1}, \boldsymbol{a}_{1}\right)_{K}+\left(\boldsymbol{a}_{2}, \boldsymbol{a}_{2}\right)_{K}+2\left(\boldsymbol{a}_{1}, \boldsymbol{a}_{2}\right)_{K}=\left(\boldsymbol{a}_{1}+\boldsymbol{a}_{2}, \boldsymbol{a}_{1}+\boldsymbol{a}_{2}\right)_{K} \geq 0 .
$$

Therefore, the wanted inequality is proved with $\alpha=\operatorname{infess}_{x \in \gamma_{1} \cup \gamma_{2}} \hat{\eta}(x)$.

We indicate with $M$ the set of indexes $i j$ corresponding to portions of fracture where we impose pressure boundary conditions, that is

$$
M:=\left\{(i, j): i, j=1,2 \text { and } \partial \gamma_{i j} \cap \partial \gamma_{i j}^{p} \neq \emptyset\right\} \quad \text { and } \quad n_{d}:=\sharp M .
$$

Theorem 4.1 (Inf-sup condition). If $n_{d}>0$, then for all $p \in Q$ there exist $a \boldsymbol{w} \in \boldsymbol{W}$ with $\boldsymbol{w} \neq \mathbf{0}$ such that

$$
\mathcal{B}(p, \boldsymbol{w}) \geq \beta\|p\|_{Q}\|\boldsymbol{w}\|_{\boldsymbol{W}},
$$

for $\beta \in \mathbb{R}^{+}$independent on $p$ and $\boldsymbol{w}$.

Proof. Given $p=\left(\hat{p}_{1}, \hat{p}_{2}, \hat{p}_{I}\right) \in Q$ we construct the following auxiliary problems. For $(i, j) \in M$ we look for a function $\phi_{i j} \in H^{2}\left(\gamma_{i j}\right)$ such that

$$
\begin{cases}-\nabla_{\boldsymbol{\tau}_{i}} \cdot\left(\nabla_{\boldsymbol{\tau}_{i}} \phi_{i j}\right)=\hat{p}_{i j} & \text { in } \gamma_{i j} \\ \frac{\partial \phi_{i j}}{\partial \boldsymbol{\tau}_{i}}=\frac{\hat{p}_{I}}{n_{d}}(-1)^{j+1}|\gamma| & \text { on } \boldsymbol{i}_{p} \\ \phi_{i j}=0 & \text { on } \partial \gamma_{i j} \cap \partial \gamma_{i j}^{p}\end{cases}
$$


with $|\gamma|=\sum_{i}\left|\gamma_{i}\right|$. While, for all other values of the indexes $i$ and $j$ we look for the $\phi_{i j}$ solution of

$$
\begin{cases}-\nabla_{\boldsymbol{\tau}_{i}} \cdot\left(\nabla_{\boldsymbol{\tau}_{i}} \phi_{i j}\right)=\hat{p}_{i j} & \text { in } \gamma_{i j}, \\ \frac{\partial \phi_{i j}}{\partial \boldsymbol{\tau}_{i}}=0 & \text { on } \partial \gamma_{i j} \cap \partial \gamma_{i j}^{\boldsymbol{u}}, \\ \phi_{i j}=0 & \text { on } \boldsymbol{i}_{p} .\end{cases}
$$

Both problems are well posed and enjoy elliptic regularity.

We consider $\boldsymbol{w}_{i j}=\nabla_{\boldsymbol{\tau}_{i}} \phi_{i j}$. We have, by construction, that the solution of (4.4) provides at the intersection point $\boldsymbol{i}_{p}$

$$
\boldsymbol{w}_{i j} \cdot \boldsymbol{\tau}_{i}=\frac{\hat{p}_{I}}{n_{d}}(-1)^{j+1}|\gamma|
$$

As for the solution of (4.5), by simple computations we derive that at $\boldsymbol{i}_{p}$

$$
\boldsymbol{w}_{i j} \cdot \boldsymbol{\tau}_{i}=-\int_{\gamma_{i j}}(-1)^{j+1} \hat{p}_{i j} d \gamma
$$

Furthermore,

$$
\mathcal{B}(p, \boldsymbol{w})=\sum_{i, j=1}^{2}\left\|\hat{p}_{i j}\right\|_{L^{2}\left(\gamma_{i j}\right)}^{2}+|\gamma| \hat{p}_{I}^{2}+\sum_{(i, j) \in M} \int_{\gamma_{i j}} \hat{p}_{i j} \hat{p}_{I} .
$$

Thanks to Young's inequality applied to the third term, we have that

$$
\mathcal{B}(p, \boldsymbol{w}) \geq \frac{1}{2}\left(\sum_{i, j=1}^{2}\left\|\hat{p}_{i j}\right\|_{L^{2}\left(\gamma_{i j}\right)}^{2}+|\gamma| \hat{p}_{I}^{2}\right) \geq c\|p\|_{Q}^{2}
$$

with $c=\frac{1}{2} \min \{1,|\gamma|\}$. Exploiting standard stability results for the solution of (4.4) and (4.5), we infer that

$$
\sum_{i=1}^{2}\left\|\boldsymbol{w}_{i j}\right\|_{L^{2}\left(\gamma_{i j}\right)}^{2}=\sum_{i=1}^{2}\left\|\nabla_{\boldsymbol{\tau}_{i}} \phi_{i j}\right\|_{L^{2}\left(\gamma_{i j}\right)}^{2} \leq C\left(\hat{p}_{I}^{2}+\sum_{i=1}^{2}\left\|\hat{p}_{i j}\right\|_{L^{2}\left(\gamma_{i j}\right)}^{2}\right) .
$$

Moreover, we have

$$
\begin{aligned}
\sum_{i=1}^{2}\left\|\nabla_{\boldsymbol{\tau}_{i}} \cdot \boldsymbol{w}_{i j}\right\|_{L^{2}\left(\gamma_{i j}\right)}^{2} & =\sum_{i=1}^{2}\left\|\nabla_{\boldsymbol{\tau}_{i}} \cdot\left(\nabla_{\boldsymbol{\tau}_{i}} \phi_{i j}\right)\right\|_{L^{2}\left(\gamma_{i j}\right)}^{2}= \\
& =\sum_{i=1}^{2}\left\|\phi_{i j}\right\|_{H^{2}\left(\gamma_{i j}\right)}^{2} \leq C\left(\hat{p}_{I}^{2}+\sum_{i=1}^{2}\left\|\hat{p}_{i j}\right\|_{L^{2}\left(\gamma_{i j}\right)}^{2}\right) .
\end{aligned}
$$

Thus, $\sum_{i j}\left\|\boldsymbol{w}_{i j}\right\|_{\boldsymbol{W}_{i j}}^{2} \lesssim\|p\|_{Q}^{2}$. Furthermore, $\llbracket \boldsymbol{w}_{i_{i}} \rrbracket_{\boldsymbol{i}_{p}}^{2} \lesssim\left\|p_{i}\right\|_{Q_{i}}^{2}+\hat{p}_{I}^{2}$ because of (4.7) and (4.6). In conclusion there exist a constant $C \in \mathbb{R}^{+}$such that $\|\boldsymbol{w}\|_{W} \leq C\|p\|_{Q}$. This result allows us to complete the proof.

Remark 4.2. The condition $n_{d} \geq 1$ in the previous proof is needed, otherwise we are not able to control the pressure $\hat{p}_{I}$. However, if all boundary conditions are imposed on the velocity we are still able to find a solution provided that the boundary velocity satisfies a global mass conservation. In this case, however, $\hat{p}_{i j} \in L^{2}(\gamma) \backslash \mathbb{R}$ and $\hat{p}_{I}$ may take any arbitrary value. 


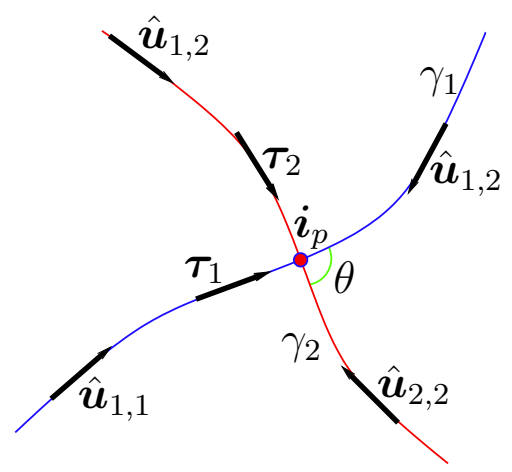

FiguRE 4. Example of an intersection with the convention of the directions for Theorem 4.3.

Lemma 4.3 (Boundedness of $\mathcal{F}$ and $\mathcal{Q}$ ). There exists $C_{1}, C_{2} \in \mathbb{R}^{+}$such that

$$
|\mathcal{F}(\boldsymbol{w})| \leq C_{1}\|\boldsymbol{w}\|_{\boldsymbol{W}} \quad \text { and } \quad|\mathcal{Q}(q)| \leq C_{2}\|q\|_{Q} \quad \forall(\boldsymbol{w}, q) \in \boldsymbol{W} \times Q .
$$

Proof. Let $(i, j) \in M$, then $\left.\left(\boldsymbol{w}_{i j} \cdot \boldsymbol{n}_{D}\right)\right|_{\partial \gamma_{i j}^{p}}$ satisfies

$$
\left|\boldsymbol{w}_{i j} \cdot \boldsymbol{n}_{D}\right| \leq\left|w_{i j}\right|\left|\boldsymbol{\tau}_{i} \cdot \boldsymbol{n}_{D}\right| \leq\left|w_{i j}\right| \leq C_{\gamma_{i j}}\left\|w_{i j}\right\|_{H^{1}\left(\gamma_{i j}\right)} \leq C_{\gamma_{i j}}\left\|\boldsymbol{w}_{i j}\right\|_{\boldsymbol{W}_{i j}} \leq C_{\gamma_{i j}}\|\boldsymbol{w}\|_{\boldsymbol{W}} .
$$

We have used the trace inequality for functions in $H^{1}$. By summing over all $(i, j) \in M$ we have

$$
|\mathcal{F}(\boldsymbol{w})| \leq \max _{i j: \partial \gamma_{i j}^{p} \neq \emptyset}\left(\left|\hat{g}_{i j}\right| C_{\gamma_{i j}}\right)\|\boldsymbol{w}\|_{\boldsymbol{W}} .
$$

Furthermore

$$
|\mathcal{Q}(q)| \leq \sum_{i=1}^{2}\left\|\hat{f}_{i}\right\|_{L^{2}\left(\gamma_{i}\right)}\left\|q_{i}\right\|_{L^{2}\left(\gamma_{i}\right)}+\left|\hat{f}_{I}\right|\left|q_{3}\right| \leq\left(\sum_{i=1}^{2}\left\|\hat{f}_{i}\right\|_{L^{2}\left(\gamma_{i}\right)}+\left|\hat{f}_{I}\right|\right)\|q\|_{Q} .
$$

Thanks to the previous results problem (4.1) is well posed [14].

We state now a maximum principle for the continuous problem (4.1). It is well know that the original problem in $\Omega_{1} \cup \Omega_{2}$ expressed by (2.3a) satisfies a maximum principle for the pressure. Namely, in the absence of the source terms $f_{i}$ and $f_{I}$ a smooth pressure solution is always within the maximal and minimal value taken at the boundary. We verify the conditions under which a similar property is enjoyed by the solution of the reduced model.

Theorem 4.3 (Maximum principle). In the case $\hat{f}_{i}=0$ and $\hat{f}_{I}=0$, if the permeability tensor $\boldsymbol{K}_{I}$ is isotropic and if the parameter $\hat{\xi}_{0}$ is such that

$$
\frac{\sin ^{2}(\theta)}{4\left(d_{1,2}^{2}+1\right)} \leq \hat{\xi}_{0} \leq \frac{d_{1,2}}{4\left(d_{1,2}^{2}+1\right)} \frac{\sin ^{2}(\theta)}{\cos (\theta)}
$$

where $d_{1,2}=d_{1} / d_{2} \leq 1$, then a maximum principle is satisfied by problem (3.11). In particular, given a smooth solution $\hat{p}$ we have that all pressures $\hat{p}_{i j}$ in $\gamma_{i j}$, as well as $\hat{p}_{I}$ are within the values taken by the pressure at boundaries $\partial \gamma_{i j} \cap \partial D$.

Proof. Let $\hat{\boldsymbol{u}}$ and $\hat{p}$ be a solution of the reduced model (3.11) with $\hat{f}_{i}=0$ and $\hat{f}_{I}=0$. Each $p_{i j}$ enjoys the maximum principle on $\gamma_{i j}$. To prove that this is the case also for the global problem it is sufficient to prove 
that: (a) the reduced pressures in the fractures at the intersection point can be expressed as convex combinations of the pressures at the external boundaries; (b) the pressure $\hat{p}_{I}$ is a convex combination of the pressures in the fractures at the intersection.

The tangent vectors $\boldsymbol{\tau}_{i}$ are continuous at $\boldsymbol{i}_{p}$. Without loss of generality we choose a frame of reference such that $\boldsymbol{\tau}_{1,2}=\left(1+m^{2}\right)^{-1}[1, \mp m]^{\top}$, with $m=\tan \theta / 2$ and $0<|m| \leq 1$, where $\theta$ is the angle between fractures as in Figure 4. Moreover, as the numbering of the fractures is arbitrary we suppose that $0<d_{1,2} \leq 1$. Since we have assumed that the permeability tensor at the intersection $\boldsymbol{K}_{I}$ is isotropic we set $\boldsymbol{K}_{I}=\eta_{i} \boldsymbol{I}$.

Let us indicate with $\bar{p}_{i j}$ the value of $p_{i j}$ at $\boldsymbol{i}_{p}$ and with $g_{i j}$ the value at the corresponding external boundary point of $\gamma_{i j}$. Then, by integrating (3.11a), we get, referring to Figure 2,

$$
\bar{p}_{i j}-g_{i j}=\hat{p}_{i}\left(L_{i j}\right)-\hat{p}_{i j}(0)=-\int_{0}^{L_{i j}} \hat{\eta}_{i} \hat{u}_{i j} \mathrm{~d} s=-\hat{u}_{i j} \int_{0}^{L_{i j}} \hat{\eta}_{i} \mathrm{~d} s,
$$

where we have denoted with $L_{i j}$ the length of the $j$-th branch of $\gamma_{i}$, and set $\hat{u}_{i j}= \pm \hat{\boldsymbol{u}}_{i} \cdot \boldsymbol{\tau}_{i}$ with the convention that $\hat{u}_{i j}$ is directed towards the intersection. Note that $\hat{u}_{i j}$ is constant because of the continuity equation and the absence of source term.

We introduce the following vectors $\boldsymbol{p}=\left[\bar{p}_{11}, \bar{p}_{12}, \bar{p}_{21}, \bar{p}_{22}\right]^{\top}, \boldsymbol{g}=\left[g_{11}, g_{12}, g_{21}, g_{22}\right]^{\top}, \boldsymbol{u}=\left[\hat{u}_{11}, \hat{u}_{12}, \hat{u}_{21}, \hat{u}_{22}\right]^{\top}$ and matrix

$$
\boldsymbol{D}=\operatorname{diag}\left\{D_{11}, D_{12}, D_{21}, D_{22}\right\} \quad \text { with } \quad D_{i j}=-\int_{0}^{L_{i j}} \hat{\eta}_{i} \mathrm{~d} s .
$$

Relation (4.8) may be rewritten as

$$
\boldsymbol{u}=\boldsymbol{D}^{-1}(\boldsymbol{g}-\boldsymbol{p})
$$

Moreover, by using the interface conditions (3.11b), which express a relationship between $\boldsymbol{u}$ and $\boldsymbol{p}$, we are able to write

$$
\boldsymbol{A p}=\boldsymbol{u} .
$$

Now we show that $\boldsymbol{A}$ is such that $\operatorname{ker}(\boldsymbol{A})=\operatorname{span}\left([1,1,1,1]^{\top}\right)$, and that $-\boldsymbol{A}$ is a Z-matrix for some values of the parameter $\hat{\xi}_{0}$. The entries of the matrix can be written as $A_{i j}=A_{N_{i j}}\left[4 \hat{\xi}_{0} \eta_{I} m^{2}\left(d_{1}^{2}+d_{2}^{2}\right)\right]^{-1}$, where the $A_{N_{i j}}$ read:

$$
\begin{aligned}
& A_{N_{11}}=A_{N_{22}}=-d_{1} d_{2}\left(\hat{\xi}_{0}\left(d_{1}^{2}+d_{2}^{2}\right)\left(1+m^{2}\right)^{2}+d_{2}^{2} m^{2}\right) \\
& A_{N_{33}}=A_{N_{44}}=-d_{2}^{3} d_{1}^{-1}\left(\hat{\xi}_{0}\left(d_{1}^{2}+d_{2}^{2}\right)\left(1+m^{2}\right)^{2}+d_{1}^{2} m^{2}\right) \\
& A_{N_{12}}=A_{N_{21}}=d_{1} d_{2}\left(\hat{\xi}_{0}\left(d_{1}^{2}+d_{2}^{2}\right)\left(1+m^{2}\right)^{2}-d_{2}^{2} m^{2}\right) \\
& A_{N_{13}}=A_{N_{31}}=A_{N_{24}}=A_{N_{24}}=d_{2}^{2}\left(\hat{\xi}_{0}\left(d_{1}^{2}+d_{2}^{2}\right)\left(1-m^{4}\right)+d_{1} d_{2} m^{2}\right) \\
& A_{N_{14}}=A_{N_{41}}=A_{N_{23}}=A_{N_{32}}=d_{2}^{2}\left(-\hat{\xi}_{0}\left(d_{1}^{2}+d_{2}^{2}\right)\left(1-m^{4}\right)+d_{1} d_{2} m^{2}\right) \\
& A_{N_{34}}=A_{N_{43}}=d_{2}^{3} d_{1}^{-1}\left(\hat{\xi}_{0}\left(d_{1}^{2}+d_{2}^{2}\right)\left(1+m^{2}\right)^{2}-d_{1}^{2} m^{2}\right)
\end{aligned}
$$

It can be verified directly that each row of the matrix sums to zero, which proves that the kernel of the matrix contains the constant vector. 
The diagonal elements of $\boldsymbol{A}$ are negative for any $\hat{\xi}_{0} \geq 0$ therefore $-\boldsymbol{A}$ is a Z-matrix if the off-diagonal elements of $\boldsymbol{A}$ are positive. It follows that the parameter $\hat{\xi}_{0}$ must satisfy the following system of inequalities

$$
\begin{aligned}
& \hat{\xi}_{0} \geq \frac{1}{d_{1,2}^{2}+1} \frac{m^{2}}{\left(1+m^{2}\right)^{2}} \quad \hat{\xi}_{0} \geq \frac{d_{1,2}^{2}}{d_{1,2}^{2}+1} \frac{m^{2}}{\left(1+m^{2}\right)^{2}} \\
& \left(1-m^{2}\right) \hat{\xi}_{0} \geq-\frac{d_{1,2}}{d_{1,2}^{2}+1} \frac{m^{2}}{\left(1+m^{2}\right)} \quad\left(1-m^{2}\right) \hat{\xi}_{0} \leq \frac{d_{1,2}}{d_{1,2}^{2}+1} \frac{m^{2}}{\left(1+m^{2}\right)}
\end{aligned}
$$

For $|m|<1$ the third inequality is satisfied for all $\hat{\xi}_{0}$ and, since $0<d_{1,2} \leq 1$, the first constraint is at least as restrictive as the second. Using the definition of $m$, the system can thus be rewritten as

$$
\frac{\sin ^{2}(\theta)}{4\left(d_{1,2}^{2}+1\right)} \leq \hat{\xi}_{0} \leq \frac{d_{1,2}}{4\left(d_{1,2}^{2}+1\right)} \frac{\sin ^{2}(\theta)}{\cos (\theta)}
$$

We have left out the case $m=1$, i.e. $\theta=\pi / 2$, but by inspecting the previous relation we see that it can be extended to this case by continuity, providing the bound $\hat{\xi}_{0} \geq \frac{1}{4\left(d_{1,2}^{2}+1\right)}$.

We highlight that the bounds depends both on the angle $\theta$ and on the ratio of the thicknesses, but not on the permeability. Combining (4.10) and (4.9) we can write

$$
\left(I+D^{-1} A\right) p=g
$$

Since $\boldsymbol{D}$ is negative and $\boldsymbol{A}$ has the aforementioned properties $\boldsymbol{I}+\boldsymbol{D}^{-1} \boldsymbol{A}$ is an M-matrix whose rows sum to one, therefore the pressures $\bar{p}_{i j}$ are convex combinations of the boundary values $\boldsymbol{g}$.

We need now to verify that $\hat{p}_{I}$ is a convex combination of the $\bar{p}_{i j}$. Summing the two interface conditions $(3.11 \mathrm{~b})$ we get $\hat{p}_{I}=\frac{1}{4} \sum_{i, j=1}^{2} \bar{p}_{i j}-\xi_{0} d_{1,2}^{-1} \eta_{i i}^{I} \sum_{i, j=1}^{2} \hat{u}_{i j}$, which becomes by using (4.10), $\hat{p}_{I}=\boldsymbol{e}^{T}\left(\frac{1}{4} \boldsymbol{I}-\xi_{0} d_{1,2}^{-1} \eta_{i i}^{I} \boldsymbol{A}\right) \boldsymbol{p}$, where $\boldsymbol{e}^{T}=[1,1,1,1]$. Because of the stated properties of $\boldsymbol{A}$, the vector $\boldsymbol{e}^{T}\left(1 / 4 \boldsymbol{I}-\xi_{0} d_{1,2}^{-1} \eta_{i i}^{I} \boldsymbol{A}\right)$ has all positive entries which sum to one, then $\hat{p}_{I}$ is a convex combination of the pressures $\boldsymbol{p}$, which completes the proof.

\section{NumERICAL DISCRETIZATION}

We still consider two fractures with a single intersection, the extension to more general cases being straightforward. We discretize each curve $\gamma_{i}, i=1,2$ with a polygonal line $\gamma_{h, i}$ with vertexes $\boldsymbol{x}_{i, k} \in \gamma_{i}$, for $k=1, \cdots, N_{h, i}$, and $\boldsymbol{x}_{i, k} \neq \mathbf{i}_{p}$. Clearly, $\boldsymbol{x}_{i, 1}$ and $\boldsymbol{x}_{i, N_{h, i}}$ correspond the ends of the curve. For the sake of notation, we indicate with the same symbol $\gamma_{h, i}$ the polygonal line and the mesh formed by the union of the line elements $l_{i, k}=\left[\boldsymbol{x}_{i, k-1}, \boldsymbol{x}_{i, k}\right]$. Moreover, we set $h_{i, k}=\left|l_{i, k}\right|$ and $h_{i}=\max _{k} h_{i, k}$, while $h=\max \left(h_{1}, h_{2}\right)$.

Let $l_{i, k}^{c}$ be the curved element on $\gamma_{i}$ with the same end points of $l_{i, k} \in \gamma_{h, i}$. If the mesh is fine enough there exists a unique mapping $\mathcal{F}_{i}: \gamma_{i} \rightarrow \gamma_{h, i}$ defined on each $l_{i, k}^{c} \in \gamma_{i}$ by

$$
\boldsymbol{x}=\mathcal{F}_{i}(\boldsymbol{y})=\boldsymbol{y}-D_{i}(\boldsymbol{y}) \boldsymbol{n}_{h, i} \quad \text { for } \quad \boldsymbol{x} \in l_{i, k} \quad \text { and } \quad \boldsymbol{y} \in l_{i, k}^{c} .
$$

Here, $\boldsymbol{n}_{h}$ is the normal vector to $\gamma_{h, i}$, which is piecewise constant in each $l_{i, k}$, and $D_{i}(\boldsymbol{y}):=\operatorname{dist}\left(\boldsymbol{y}, \gamma_{h, i}\right)$. The collection of the curved elements $l_{i, k}^{c}$ will be denoted by $\gamma_{h, i}^{c}$, which geometrically coincides with $\gamma_{i}$. Because of the assumptions made on the regularity of $\gamma_{i}$, and in particular the boundedness on the curvature, we have that $|D(\boldsymbol{y})|=\mathcal{O}\left(h^{2}\right)$ for all $\boldsymbol{y} \in \gamma_{i}$. If we indicate with $\dot{D}_{i}$ the rate of variation of $D_{i}(\boldsymbol{y})$ along $\gamma_{h, i}$, we may note that the arc length measures on $\gamma_{i}$ and $\gamma_{h, i}$ satisfy

$$
\mathrm{d} \gamma_{i}=\left(1+\dot{D}_{i}^{2}\right)^{1 / 2} \mathrm{~d} \gamma_{h, i} .
$$


We assume that the mesh is fine enough so that $\dot{D}_{i}=\mathcal{O}(h)$ and, in particular, we have $\dot{D}_{i} \in L^{\infty}\left(\gamma_{h, i}\right)$. By properly selecting the orientation of the curves we have the useful relations

$$
\boldsymbol{n}_{h, i} \cdot \boldsymbol{\tau}_{i}=\dot{D}_{i}\left(1+\dot{D}_{i}^{2}\right)^{-1 / 2} \quad \text { and } \quad \boldsymbol{\tau}_{i} \cdot \boldsymbol{\tau}_{h, i}=\left(1+\dot{D}_{i}^{2}\right)^{-1 / 2},
$$

where $\boldsymbol{\tau}_{h, i}$ is the (piecewise constant) tangent vector of $\gamma_{h, i}$. Furthermore, we have that $\left(1+\dot{D}_{i}^{2}\right)^{-1 / 2}=1+\mathcal{O}(h)$. Let now $f: \gamma_{h, i} \rightarrow \mathbb{R}$, we consider the transformations $\mathcal{P}_{i}$ given by

$$
f^{c}=\mathcal{P}_{i} f=f \circ \mathcal{F}_{i} .
$$

Clearly, $f^{c}: \gamma_{i} \rightarrow \mathbb{R}$.

For a vector function $\boldsymbol{v}: \gamma_{h, i} \rightarrow \mathbb{R}^{2}$ aligned with $\gamma_{h, i}$, i.e. $\boldsymbol{v}=v \boldsymbol{\tau}_{h, i}$, we consider instead the transformation $\mathcal{P}_{i}$ given by

$$
\boldsymbol{v}^{c}=\mathcal{P}_{i} \boldsymbol{v}=\left(\boldsymbol{v} \circ \mathcal{F}_{i}\right) \cdot \boldsymbol{\tau}_{h, i} \boldsymbol{\tau}_{i}=\left(\boldsymbol{v} \circ \mathcal{F}_{i}\right) \boldsymbol{\tau}_{h, i} \otimes \boldsymbol{\tau}_{i} .
$$

Lemma 5.1. Transformation $\mathcal{P}_{i}$ is an isomorphism between $H^{1}\left(\gamma_{h, i}\right)$ and $H^{1}\left(\gamma_{i}\right)$, while transformation $\mathcal{P}_{i}$ is an isomorphism between $\boldsymbol{H}_{\mathrm{div}}\left(\gamma_{h, i}\right)$ and $\boldsymbol{H}_{\mathrm{div}}\left(\gamma_{i}\right)$. Furthermore,

$$
\int_{\gamma_{i}} \nabla_{\boldsymbol{\tau}_{i}} \cdot \boldsymbol{v}^{c} q^{c} \mathrm{~d} \gamma=\int_{\gamma_{h, i}} \nabla_{\boldsymbol{\tau}_{h, i}} \cdot \boldsymbol{v} q \mathrm{~d} \gamma
$$

for all $q \in L^{2}\left(\gamma_{h, i}\right), \boldsymbol{v} \in \boldsymbol{H}_{\mathrm{div}}\left(\gamma_{h, i}\right)$ with $q^{c}=\mathcal{P}_{i} q$ and $\boldsymbol{v}^{c}=\mathcal{P}_{i} \boldsymbol{v}$, respectively. Moreover, for each element $l_{h, i}$ of $\gamma_{h, i}$

$$
\left|\nabla_{\boldsymbol{\tau}_{h, i}} \cdot \boldsymbol{v}\right|_{\boldsymbol{H}^{1}\left(l_{h, i}\right)}=\left\|\nabla_{\boldsymbol{\tau}_{h, i}} \nabla_{\boldsymbol{\tau}_{h, i}} \cdot \boldsymbol{v}\right\|_{L^{2}\left(l_{h, i}\right)} \lesssim\left|\nabla_{\boldsymbol{\tau}_{i}} \cdot \boldsymbol{v}^{c}\right|_{\boldsymbol{H}^{1}\left(l_{h, i}^{c}\right)}+h\left\|\nabla_{\boldsymbol{\tau}_{i}} \cdot \boldsymbol{v}^{c}\right\|_{L^{2}\left(l_{h, i}^{c}\right)} .
$$

Proof. By standard integration rules

$$
\left\|f^{c}\right\|_{L^{2}\left(\gamma_{i}\right)}^{2}=\int_{\gamma_{i}}\left(f^{c}\right)^{2} \mathrm{~d} \gamma=\int_{\gamma_{h, i}}\left(1+\dot{D}_{i}^{2}\right)^{1 / 2} f^{2} \mathrm{~d} \gamma
$$

Thus,

$$
\|f\|_{L^{2}\left(\gamma_{h, i}\right)}^{2} \leq\left\|f^{c}\right\|_{L^{2}\left(\gamma_{i}\right)}^{2} \leq C_{h}\|f\|_{L^{2}\left(\gamma_{h, i}\right)}^{2},
$$

with $C_{h}=1+\mathcal{O}(h) \geq 1$. By the same technique we prove that

$$
\|\boldsymbol{v}\|_{L^{2}\left(\gamma_{h, i}\right)}^{2} \leq\left\|\boldsymbol{v}^{c}\right\|_{L^{2}\left(\gamma_{i}\right)}^{2} \leq C_{h}\|\boldsymbol{v}\|_{L^{2}\left(\gamma_{h, i}\right)}^{2} .
$$

We now note that if $s$ and $t$ denote the arc length coordinates along $\gamma_{i}$ and $\gamma_{h, i}$, respectively, for a vector function $\boldsymbol{v}=\boldsymbol{v} \boldsymbol{\tau}_{h, i}$ aligned along $\gamma_{h, i}$ we have the identities

$$
\nabla_{\boldsymbol{\tau}_{h, i}} \cdot \boldsymbol{v}=\frac{\mathrm{d} v}{\mathrm{~d} t} \quad \text { and } \quad \nabla_{\boldsymbol{\tau}_{i}} \cdot \boldsymbol{v}^{c}=\frac{\mathrm{d} v^{c}}{\mathrm{~d} s} .
$$

Thus,

$$
\nabla_{\boldsymbol{\tau}_{i}} \cdot \boldsymbol{v}^{c}=\frac{\mathrm{d} v \circ \mathcal{F}_{i}}{\mathrm{~d} s}=\frac{\mathrm{d} v}{\mathrm{~d} t} \frac{\mathrm{d} t}{\mathrm{~d} s}=\left(1+\dot{D}_{i}^{2}\right)^{-1 / 2} \nabla_{\boldsymbol{\tau}_{h, i}} \cdot \boldsymbol{v},
$$

since $\mathrm{d} s / \mathrm{d} t=\left(1+\dot{D}_{i}^{2}\right)^{1 / 2}$. Consequently,

$$
c_{h}\left\|\nabla_{\boldsymbol{\tau}_{h, i}} \cdot \boldsymbol{v}\right\|_{L^{2}\left(\gamma_{h, i}\right)} \leq\left\|\nabla_{\boldsymbol{\tau}_{i}} \cdot \boldsymbol{v}^{c}\right\|_{L^{2}\left(\gamma_{i}\right)} \leq\left\|\nabla_{\boldsymbol{\tau}_{h, i}} \cdot \boldsymbol{v}\right\|_{L^{2}\left(\gamma_{h, i}\right)},
$$


where $c_{h}$ is a positive constant that behaves as $c_{h}=1-\mathcal{O}(h)$. Combining this last result with (5.5) we get

$$
c_{h}\|\boldsymbol{v}\|_{\boldsymbol{H}_{\mathrm{div}}\left(\gamma_{h, i}\right)} \leq\left\|\boldsymbol{v}^{c}\right\|_{\boldsymbol{H}_{\mathrm{div}}\left(\gamma_{i}\right)} \leq C_{h}\|\boldsymbol{v}\|_{\boldsymbol{H}_{\mathrm{div}}\left(\gamma_{h, i}\right)} .
$$

Analogously,

$$
\nabla_{\boldsymbol{\tau}_{i}} f^{c}=\frac{\mathrm{d} f^{c}}{\mathrm{~d} s} \boldsymbol{\tau}_{i} \text { and } \quad \nabla_{\boldsymbol{\tau}_{h, i}} f=\frac{\mathrm{d} f}{\mathrm{~d} t} \boldsymbol{\tau}_{h, i}
$$

Thus,

$$
\nabla_{\boldsymbol{\tau}_{i}} f^{c}=\frac{\mathrm{d} f \circ \mathcal{F}_{i}}{\mathrm{~d} t} \frac{\mathrm{d} t}{\mathrm{~d} s} \boldsymbol{\tau}_{i}=\left(1+\dot{D}_{i}^{2}\right)^{-1 / 2} \nabla_{\boldsymbol{\tau}_{h, i}} f \cdot \boldsymbol{\tau}_{h, i} \boldsymbol{\tau}_{i} .
$$

Taking the $L^{2}$ norm and applying the definition of the $H^{1}\left(\gamma_{h, i}\right)$ semi-norm we obtain

$$
c_{h}|f|_{H^{1}\left(\gamma_{h, i}\right)} \leq\left|f^{c}\right|_{H^{1}\left(\gamma_{i}\right)} \leq C_{h}|f|_{H^{1}\left(\gamma_{h, i}\right)} .
$$

As for (5.4) we use again the parametric representation to note that on each element $l_{h, i}$

$$
\begin{aligned}
\nabla_{\boldsymbol{\tau}_{i}} \nabla_{\boldsymbol{\tau}_{i}} \cdot \boldsymbol{v}_{h, i}^{c} & =\frac{\mathrm{d}}{\mathrm{d} s}\left(\nabla_{\boldsymbol{\tau}_{i}} \cdot \boldsymbol{v}_{h, i}^{c}\right) \boldsymbol{\tau}_{i}=\frac{\mathrm{d}}{\mathrm{d} t}\left[\left(1+\dot{D}^{2}\right)^{-1 / 2} \nabla_{\boldsymbol{\tau}_{h, i}} \cdot \boldsymbol{v}_{h, i}\right] \boldsymbol{\tau}_{i} \\
& =\left(1+\dot{D}^{2}\right)^{-1 / 2} \frac{\mathrm{d}^{2}}{\mathrm{~d} t^{2}} v_{h, i} \boldsymbol{\tau}_{i}-\frac{\dot{D} \ddot{D}}{\left(1+\dot{D}^{2}\right)^{2}} \frac{\mathrm{d}}{\mathrm{d} t} v_{h, i} \boldsymbol{\tau}_{i} \\
& =\left(1+\dot{D}^{2}\right)^{-1 / 2} \boldsymbol{\tau}_{i} \otimes \boldsymbol{\tau}_{h, i} \nabla_{\boldsymbol{\tau}_{h, i}} \nabla_{\boldsymbol{\tau}_{h, i}} \cdot \boldsymbol{v}_{h, i}-\frac{\dot{D} \ddot{D}}{\left(1+\dot{D}^{2}\right)} \nabla_{\boldsymbol{\tau}_{i}} \cdot \boldsymbol{v}_{h, i}^{c} \boldsymbol{\tau}_{i}
\end{aligned}
$$

where it is understood that quantities are computed on corresponding points on $l_{h, i}$ and $l_{h, i}^{c}$, and we have used (5.7) and (5.8). By taking the $L^{2}$ norm and using the fact that $\dot{D}=\mathcal{O}(h)$ we obtain the wanted result. Finally, relation (5.3) is readily proved using (5.8) and applying the usual integration rules.

We are now in the position of setting up our discrete spaces. We start by defining, for $i=1,2$,

$$
\mathbb{R T}_{0}\left(\gamma_{h, i}\right)=\left\{\boldsymbol{w} \in \boldsymbol{H}_{\mathrm{div}}\left(\gamma_{h, i}\right): \boldsymbol{w} \in \mathbb{P}_{1}(l), \forall l \in \gamma_{h, i}, \boldsymbol{w}=w \boldsymbol{\tau}_{h, i}, w \in C^{0}\left(\gamma_{h, i}\right)\right\} .
$$

Note that despite the fact that $\boldsymbol{\tau}_{h, i}$ is only piecewise continuous the tangential component of elements of $\mathbb{R T}_{0}\left(\gamma_{h, i}\right)$ is continuous. We also remind that, since we are treating problems on a one dimensional manifold, elements of $\boldsymbol{H}_{\mathrm{div}}\left(\gamma_{h, i}\right)$ have tangential component in $H^{1}$ and thus admit a continuous representative. The degrees of freedom on $\mathbb{R T}_{0}\left(\gamma_{h, i}\right)$ are indeed the values of $w=\boldsymbol{w} \cdot \boldsymbol{\tau}_{h, i}$ at the mesh nodes. Correspondingly we have a set of basis function which we indicate as $\left\{\boldsymbol{\psi}_{i, k}, k=1, \ldots, N_{h, i}\right\}$.

To account for the discontinuity at the intersection we consider the points $\widehat{\boldsymbol{i}}_{p}=\mathcal{F}_{i}\left(\boldsymbol{i}_{p}\right)$ projection of $\boldsymbol{i}_{p}$ on $\gamma_{h, i}$ and we enrich the space using the XFEM [22] methodology.

More precisely, let $\mathcal{C}_{h, i}=\left[\boldsymbol{x}_{i, k-1}, \boldsymbol{x}_{i, k}\right]$ be the element crossed by the projected intersection point (see Fig. 5 ) and $\chi_{i, 1}$ and $\chi_{i, 2}$ the characteristic functions of the sub-elements $l_{i, 1}^{X}=\left[\boldsymbol{x}_{i, k-1}, \widehat{\boldsymbol{i}}_{p}\right]$ and $l_{i, 2}^{X}=\left[\widehat{\boldsymbol{i}}_{p}, \boldsymbol{x}_{i, k}\right]$, respectively. We consider the space

$$
\boldsymbol{E}_{u}\left(\gamma_{h, i}\right):=\left\{\boldsymbol{v}_{h, i}: \boldsymbol{v}_{h, i}=v_{i, 1}^{*} \boldsymbol{\psi}_{i, k} \chi_{i, 1}+v_{i, 2}^{*} \boldsymbol{\psi}_{i, k-1} \chi_{i, 2}\right\} .
$$

The spaces $\mathbb{R T}_{0}$ and $\boldsymbol{E}_{u}$ have been defined on $\gamma_{h, i}$, we can then project them on the curve and account for essential boundary conditions by defining

$$
\boldsymbol{W}_{h, i}^{c}:=\mathcal{P}_{i}\left(\mathbb{R T}_{0}\left(\gamma_{h, i}\right) \oplus \boldsymbol{E}_{p}\left(\gamma_{h, i}\right)\right) \cap \boldsymbol{W}_{i} .
$$

Correspondingly, $\boldsymbol{W}_{h, i}=\mathcal{P}_{i}^{-1}\left(\boldsymbol{W}_{h, i}^{c}\right)$. By construction, $\boldsymbol{W}_{h, i} \subset \mathbb{R}_{0}\left(\gamma_{h, i}\right) \oplus \boldsymbol{E}_{p}\left(\gamma_{h, i}\right)$.

We can define $\gamma_{h, i j}=\left(\hat{\gamma}_{i j} \backslash \mathcal{C}_{h, i}\right) \cup l_{i, j}^{X}$, where $\hat{\gamma}_{i j}=\left\{l \in \gamma_{h, i}: \mathcal{F}^{-1}(l) \cap \gamma_{i j} \neq \emptyset\right\}$. 


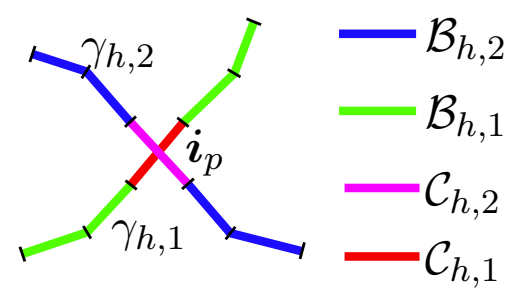

FiguRE 5. Subdivision of $\gamma_{h, i}=\mathcal{C}_{h, i} \cup \mathcal{B}_{h, i}$.

Remark 5.1. The points $\mathcal{F}_{i}\left(\boldsymbol{i}_{p}\right)$ are in general different, unless fractures $\gamma_{i}$ are straight lines, and $\left\|\mathcal{F}_{1}\left(\boldsymbol{i}_{p}\right)-\mathcal{F}_{2}\left(\boldsymbol{i}_{p}\right)\right\|=\mathcal{O}\left(h^{2}\right)$ as $h \rightarrow 0$. However, in most practical situations fractures are almost straight and for a sufficiently fine mesh the distance of the two projections is rather small. For this reason, and for the sake of notation, we have used a unique symbol, $\widehat{\boldsymbol{i}}_{p}$ for both projected intersection points.

For the pressure we proceed by setting

$$
Q_{h, i}:=\left\{q \in L^{2}\left(\gamma_{h, i}\right):\left.q\right|_{l}=q_{i l}, \forall l \in \gamma_{h, i} \backslash \mathcal{C}_{h, i},\left.q\right|_{\mathcal{C}_{h, i}}=q_{i 1}^{*} \chi_{i, 1}+q_{i 2}^{*} \chi_{i, 2}\right\},
$$

which is the extended space of piecewise constant functions on $\gamma_{h, i}$, and we lift it to $\gamma_{i}$, by defining $Q_{h, i}^{c}:=$ $\mathcal{P}_{i}\left(Q_{h, i}\right)$. The space $Q_{h, i}^{c}$ is in fact made by piecewise constant functions on the curved mesh $\gamma_{h, i}^{c}$. By construction, both $\boldsymbol{W}_{h, i}$ and $Q_{h, i}$ are broken spaces, i.e.

$$
\boldsymbol{W}_{h, i}=\boldsymbol{W}_{h, i 1} \times \boldsymbol{W}_{h, i 2} \quad \text { and } \quad Q_{h, i}=Q_{h, i 1} \times Q_{h, i 2},
$$

where $\boldsymbol{W}_{h, i j}$ is the restriction of $\boldsymbol{W}_{h, i}$ on $\gamma_{h, i j}$, and we have that $\boldsymbol{W}_{h, i j} \subset \boldsymbol{H}_{\text {div }}\left(\gamma_{h, i j}\right)$. Consequently, also $\boldsymbol{W}_{h, i}^{c}$ and $Q_{h, i}^{c}$ can be written as

$$
\boldsymbol{W}_{h, i}^{c}=\boldsymbol{W}_{h, i 1}^{c} \times \boldsymbol{W}_{h, i 2}^{c} \quad \text { and } \quad Q_{h, i}^{c}=Q_{h, i 1}^{c} \times Q_{h, i 2}^{c},
$$

with $\boldsymbol{W}_{h, i j}^{c} \subset \boldsymbol{W}_{i j}$ and $Q_{h, i j}^{c} \subset Q_{i j}$. Thus $\boldsymbol{W}_{h, i}^{c} \subset \boldsymbol{W}_{i}$ and $Q_{h, i}^{c} \subset Q_{i}$. We define $\boldsymbol{W}_{h}^{c}:=\boldsymbol{W}_{h, 1}^{c} \times \boldsymbol{W}_{h, 2}^{c}$ and $Q_{h}^{c}=Q_{h, 1}^{c} \times Q_{h, 2}^{c} \times \mathbb{R}$, and analogously $\boldsymbol{W}_{h}$ and $Q_{h}$.

We can now write the discrete weak formulation of the problem (3.11) as: find $\left(\hat{\boldsymbol{u}}_{h}^{c}, \hat{p}_{h}^{c}\right) \in \boldsymbol{W}_{h}^{c} \times Q_{h}^{c}$ such that

$$
\begin{cases}\mathcal{A}\left(\hat{\boldsymbol{u}}_{h}^{c}, \boldsymbol{w}_{h}^{c}\right)+\mathcal{B}\left(\hat{p}_{h}^{c}, \boldsymbol{w}_{h}^{c}\right)=\mathcal{F}\left(\boldsymbol{w}_{h}^{c}\right) & \forall \boldsymbol{w}_{h}^{c} \in \boldsymbol{W}_{h}^{c} \\ \mathcal{B}\left(q_{h}^{c}, \hat{\boldsymbol{u}}_{h}^{c}\right)=\mathcal{Q}\left(q_{h}^{c}\right) & \forall q_{h}^{c} \in Q_{h}^{c} .\end{cases}
$$

We introduce the following weighted $L^{2}$ norm on functions of $L^{2}\left(\gamma_{h, i}\right)$

$$
\|w\|_{L_{h}^{2}\left(\gamma_{h, i}\right)}^{2}=\int_{\gamma_{h, i}}\left(1+\dot{D}^{2}\right)^{-1 / 2} w^{2} \mathrm{~d} \gamma
$$

which is equivalent to the natural $L^{2}$ norm thanks to Lemma 5.1.

We define now the following problem on the polygonal lines approximating the fractures: find $\left(\hat{\boldsymbol{u}}_{h}, \hat{p}_{h}\right) \in$ $\boldsymbol{W}_{h} \times Q_{h}$ such that

$$
\begin{cases}\mathcal{A}_{h}\left(\hat{\boldsymbol{u}}_{h}, \boldsymbol{w}_{h}\right)+\mathcal{B}_{h}\left(\hat{p}_{h}, \boldsymbol{w}_{h}\right)=\mathcal{F}_{h}\left(\boldsymbol{w}_{h}\right) & \forall \boldsymbol{w}_{h} \in \boldsymbol{W}_{h} \\ \mathcal{B}_{h}\left(q_{h}, \hat{\boldsymbol{u}}_{h}\right)=\mathcal{Q}_{h}\left(q_{h}\right) & \forall q_{h} \in Q_{h},\end{cases}
$$


where

$$
\begin{aligned}
\mathcal{A}_{h}\left(\boldsymbol{u}_{h}, \boldsymbol{w}_{h}\right) & :=\sum_{i=1}^{2} a_{h, i}\left(\boldsymbol{u}_{h, i}, \boldsymbol{w}_{h, i}\right)+\sum_{\substack{i, j=1 \\
i \neq j}}^{2} \eta_{i j}^{I}\left\{\{ \boldsymbol { u } _ { h , j } \cdot \boldsymbol { \tau } _ { h , j } \} _ { \hat { \boldsymbol { i } } _ { p } } \left\{\left\{\boldsymbol{w}_{h, i} \cdot \boldsymbol{\tau}_{h, i}\right\}_{\hat{\boldsymbol{i}}_{p}},\right.\right. \\
\mathcal{B}_{h}\left(q_{h}, \boldsymbol{w}_{h}\right) & :=-\sum_{i=1}^{2}\left(q_{h, i}, \nabla_{\boldsymbol{\tau}_{h, i}} \cdot \boldsymbol{w}_{h, i}\right)_{L^{2}\left(\gamma_{h, i}\right)}+q_{3} \llbracket \boldsymbol{w}_{h, i} \cdot \boldsymbol{\tau}_{h, i} \rrbracket_{\hat{\boldsymbol{i}}_{p}}, \\
\mathcal{F}_{h}(\boldsymbol{w}) & :=\sum_{i j: \partial \gamma_{i j}^{p} \neq \emptyset}-\hat{g}_{i} \boldsymbol{w}_{h, i} \cdot \mathcal{P}_{i}^{-1} \boldsymbol{n}_{D}, \\
\mathcal{Q}_{h}(q) & :=\sum_{i=1}^{2}-\left(\hat{f}_{i} \circ \mathcal{F}_{i}^{-1}, q_{h, i}\right)_{L_{h}^{2}\left(\gamma_{i}\right)}+\hat{f}_{I} q_{3} .
\end{aligned}
$$

Here, the bilinear forms $a_{h, i}$ are defined as

$$
\begin{aligned}
a_{h, i}(\boldsymbol{u}, \boldsymbol{w}):= & \left(\hat{\eta}_{i} \boldsymbol{u}, \boldsymbol{w}\right)_{L_{h}^{2}\left(\gamma_{i}\right)}+\sum_{i=1}^{2} \eta^{I} \frac{d_{j}}{d_{i}}\left(\hat{\xi}_{0} \llbracket \boldsymbol{u}_{h, i} \cdot \boldsymbol{\tau}_{h, i} \rrbracket_{\widehat{\boldsymbol{i}}_{p}} \llbracket \boldsymbol{w}_{h, i} \cdot \boldsymbol{\tau}_{h, i} \rrbracket_{\widehat{\boldsymbol{i}}_{p}}\right. \\
& +\left\{\left[\boldsymbol{u}_{h, i} \cdot \boldsymbol{\tau}_{h, i}\right\}_{\hat{\boldsymbol{i}}_{p}}\left\{\left\{\boldsymbol{w}_{h, i} \cdot \boldsymbol{\tau}_{h, i}\right\}_{\widehat{\boldsymbol{i}}_{p}}\right) \text { with } j \neq i .\right.
\end{aligned}
$$

Lemma 5.2. Problem (5.12) is equivalent to (5.14) in the sense that if $\hat{\boldsymbol{u}}_{h}=\left(\boldsymbol{u}_{h, 1}, \boldsymbol{u}_{h, 2}\right) \in \boldsymbol{W}_{h}$ and $\hat{p}_{h}=$ $\left(q_{1}, q_{2}, q_{I}\right) \in Q_{h}$ is a solution of (5.14) then the projections $\left(\mathcal{P}_{1} \boldsymbol{u}_{h, 1}, \mathcal{P}_{2} \boldsymbol{u}_{h, 2}\right) \in \boldsymbol{W}_{h}^{c}$ and $\left(\mathcal{P}_{1} q_{1}, \mathcal{P}_{2} q_{2}, q_{I}\right) \in Q_{h}^{c}$ are a solution of (5.12). Vice versa, if $\hat{\boldsymbol{u}}_{h}^{c}=\left(\boldsymbol{u}_{h, 1}^{c}, \boldsymbol{u}_{h, 2}^{c}\right) \in \boldsymbol{W}_{h}^{c}$ and $\hat{p}_{h}^{c}=\left(q_{1}^{c}, q_{2}^{c}, q_{I}\right) \in Q_{h}^{c}$ is a solution of (5.14) then $\left(\mathcal{P}_{1}^{-1} \boldsymbol{u}_{h, 1}^{c}, \mathcal{P}_{2}^{-1} \boldsymbol{u}_{h, 2}^{c}\right) \in \boldsymbol{W}_{h}$ and $\left(\mathcal{P}_{1}^{-1} q_{1}, \mathcal{P}_{2}^{-1} q_{2}, q_{I}\right) \in Q_{h}$ are a solution of (5.12).

Proof. It is sufficient to apply the definition of the discrete spaces and of the transformations $\mathcal{P}_{i}$ and $\mathcal{P}_{i}$, together with (5.3), (5.13) and apply Lemma 5.1.

Theorem 5.2 (Well posedness of the discrete problem). Under the same conditions of Theorem 4.1, problem (5.12) is well posed.

Proof. We tackle problem (5.12) by considering the equivalent problem (5.14) instead. First of all we note that thanks to Lemma 5.1 all bilinear forms and functionals in (5.14) are bounded, since we have already demonstrated the boundedness of the ones used in (5.12). We now note that $\boldsymbol{W}_{h, i j}$ and $Q_{h, i j}$ do in fact define a one dimensional $\mathbb{R T}_{0}$ and a piecewise constant finite element space, respectively. Therefore we can define a standard interpolation $\Pi_{i j}: \boldsymbol{H}_{\mathrm{div}}\left(\gamma_{h, i j}\right) \rightarrow \boldsymbol{W}_{h, i j}$ and projection operators $\pi_{i j}: L^{2}\left(\gamma_{h, i j}\right) \rightarrow Q_{h, i j}$. It is known that in one dimension the two operator commute with the tangential divergence, i.e. $\nabla_{\tau_{h, i}} \cdot \Pi_{i j} \boldsymbol{v}=\pi_{i j} \nabla_{\tau_{h, i}} \boldsymbol{v}$, for all $\boldsymbol{v} \in \boldsymbol{H}_{\mathrm{div}}\left(\gamma_{h, i j}\right)$.

We can then repeat the same steps of Theorem 4.1 on problem (5.14), where now we take as velocity field associated to a given $q_{h, i j} \in Q_{h, i j}$ the quantity $\boldsymbol{v}_{h, i j}=\Pi_{i j} \nabla_{\tau_{h, i}} \phi_{i j}$ to prove the inf-sup stability of $\mathcal{B}_{h}$.

Theorem 5.3 (Maximum principle). The maximum principle is satisfied by problem (5.12) under the same conditions of Theorem 4.3.

Proof. Again we refer first to the equivalent problem (5.14). In the absence of source term the second equation implies that $\nabla_{\boldsymbol{\tau}_{h, i}} \boldsymbol{u}_{h, i}=0$ on each element of $\gamma_{h, i j}$. Therefore $\boldsymbol{u}_{h, i j}=u_{h, i j} \boldsymbol{\tau}_{h, i}$ is constant. For any couple $i, j$ we take as test function $\boldsymbol{w}_{h}$ the function such that $\boldsymbol{w}_{h, i j} \cdot \boldsymbol{\tau}_{h, i j}=1$ on all vertexes $\boldsymbol{x}_{k}$ internal to $\hat{\gamma}_{h, i j}$ and zero at all other nodes and on the intersection point $\widehat{\boldsymbol{i}}_{p}$. Then, by simple computations, the first equation of (5.14) 
gives the following relation for the pressures $\hat{p}_{h, i j, 1}$ and $\hat{p}_{h, i j, N_{i j}}$ at the first and last element $l_{h, i j, 1}$ and $l_{h, i j, N_{i j}}$ of $\gamma_{h, i j}$,

$$
\hat{p}_{h, i j, N_{h}}-\hat{p}_{h, i j, 1}=-\boldsymbol{u}_{h, i j} \int_{\hat{\gamma}_{h, i j}} \hat{\eta}_{i} \boldsymbol{w}_{h, i j} \cdot \boldsymbol{\tau}_{h, i j} \mathrm{~d} x
$$

Since $\boldsymbol{w}_{h, i j} \cdot \boldsymbol{\tau}_{h, i j}$ is not negative, $\hat{p}_{h, i j}$ is varying monotonically at the interior nodes of $\hat{\gamma}_{h, i j}$. Thus, also in this case we are left to prove the same conditions (a) and (b) stated in Theorem 4.3, where now $\boldsymbol{g}=$ $\left[\hat{p}_{h, 11,1}, \hat{p}_{h, 12,1}, \hat{p}_{h, 21,1}, \hat{p}_{h, 2,1}\right]^{\top}$ and $\boldsymbol{p}=\left[\hat{p}_{h, 11, N_{h}}, \hat{p}_{h, 12, N_{h}}, \hat{p}_{h, 21, N_{h}}, \hat{p}_{h, 22, N_{h}}\right]^{\top}$. Since the interface conditions at the intersection are unchanged from the continuous case we can repeat the same argument of the cited theorem to conclude the proof for what concerns the solution of (5.14).

As for the solution of (5.12), it is sufficient to recall Lemma 5.2 and note that the elemental values of the pressure are unchanged in the two problems and that the transformation $\mathcal{P}_{i}$ maintains monotonicity.

Theorem 5.4 (Convergence). Let $(\hat{\boldsymbol{u}}, \hat{p})$ be solution of (4.1) with $\hat{\boldsymbol{u}}_{i} \in \boldsymbol{H}^{2}\left(\gamma_{i 1} \cup \gamma_{i 2}\right)$ and $\hat{p}_{i} \in H^{1}\left(\gamma_{i 1} \cup \gamma_{i 2}\right)$ and $\left(\hat{\boldsymbol{u}}_{h}^{c}, \hat{p}_{h}^{c}\right)$ be solution of $(5.12)$, then

$$
\left\|\hat{\boldsymbol{u}}-\hat{\boldsymbol{u}}_{h}^{c}\right\|_{\boldsymbol{W}}+\left\|\hat{p}-\hat{p}_{h}^{c}\right\|_{Q} \leq C h\left(\|\hat{\boldsymbol{u}}\|_{\mathcal{W}}+|\hat{p}|_{\mathcal{Q}}\right),
$$

where

$$
\|\hat{\boldsymbol{u}}\|_{\mathcal{W}}^{2}=\sum_{i, j=1}^{2}\|\hat{\boldsymbol{u}}\|_{\boldsymbol{H}^{2}\left(\gamma_{i j}\right)}^{2} \quad \text { and } \quad|\hat{p}|_{\mathcal{Q}}^{2}=\sum_{i, j=1}^{2}|\hat{p}|_{H^{1}\left(\gamma_{i j}\right)}^{2}
$$

Proof. By standard results of saddle point problems $[14,16]$ we have that there exist a constant $C$ independent from $h$ such that

$$
\left\|\hat{\boldsymbol{u}}-\hat{\boldsymbol{u}}_{h}^{c}\right\|_{\boldsymbol{W}}+\left\|\hat{p}-\hat{p}_{h}^{c}\right\|_{Q} \lesssim\left(\inf _{\boldsymbol{w}_{h}^{c} \in \boldsymbol{W}_{h}^{c}}\left\|\hat{\boldsymbol{u}}-\boldsymbol{w}_{h}^{c}\right\|_{\boldsymbol{W}}+\inf _{q_{h}^{c} \in Q_{h}^{c}}\left\|\hat{p}-q_{h}^{c}\right\|_{Q}\right) .
$$

We set $\mathcal{P}$ to be the composition of the operators $\mathcal{P}_{i}$ operating on each portion $\gamma_{i j}$ of the fractures. That is, for a $\mathbf{w}_{h}^{c} \in \boldsymbol{W}$ we have that $\mathcal{P}^{-1} \mathbf{w}_{h}^{c}=\prod_{i, j=1}^{2} \mathcal{P}_{i}^{-1} \mathbf{w}_{i j}^{c} \in \boldsymbol{W}_{h}$. Because of Lemma 5.1 we have

$$
\left\|\hat{\boldsymbol{u}}-\boldsymbol{w}_{h}^{c}\right\|_{\boldsymbol{W}} \lesssim\left\|\mathcal{P}^{-1} \hat{\boldsymbol{u}}-\boldsymbol{w}_{h}\right\|_{\boldsymbol{W}_{h}}
$$

where $\boldsymbol{w}_{h}=\mathcal{P}^{-1} \boldsymbol{w}_{h}^{c} \in \boldsymbol{W}_{h}$.

To carry on with the proof, we cannot use the properties of Raviart-Thomas interpolation directly because of the extended finite elements. We may however operate in the way indicated in [15], Theorem 4.8, which is in turn based on what introduced in [9], Theorem 3. It is a standard technique in the analysis of the XFEM method and it consists in using a continuous extension $E_{i j}^{2}: \boldsymbol{H}^{2}\left(\gamma_{i j}\right) \rightarrow \boldsymbol{H}^{2}\left(\gamma_{i}\right)$ in order to be able to bound the terms resulting from the integration in the cut elements.

A minor technical difficulty in our case is that even if (by the hypothesis of the Theorem) $\hat{\boldsymbol{u}}_{i j}$ belongs to $\boldsymbol{H}^{2}\left(\gamma_{i j}\right), \mathcal{P}^{-1} \hat{\boldsymbol{u}}_{i j}$ in general does not. Therefore, we cannot apply a $\boldsymbol{H}^{2}$ extension to $\mathcal{P}^{-1} \hat{\boldsymbol{u}}_{i j}$ but only to $\hat{\boldsymbol{u}}_{i j}$. We thus define an extended $\mathbb{R} \mathbb{T}_{0}$ interpolant $\Pi_{i}^{*}$ acting on each $\hat{\boldsymbol{u}}_{i j}$ as $\Pi_{i}^{*} \hat{\boldsymbol{u}}_{i j}=\Pi_{i} \mathcal{P}^{-1} E_{i j}^{2} \hat{\boldsymbol{u}}_{i j}, \Pi_{i}$ being the standard $\mathbb{R T}_{0}$ interpolant on $\gamma_{h, i}$. Clearly, $\left.\Pi_{i}^{*} \hat{\boldsymbol{u}}_{i j}\right|_{\gamma_{i j}} \in \boldsymbol{W}_{h, i j}$, and is in fact extended to the whole $\gamma_{h, i}$. We exploit now the fact that if the curves $\gamma_{i}$ are sufficiently regular, $\left.\mathcal{P}^{-1} \hat{\boldsymbol{u}}_{i j}\right|_{l} \in \boldsymbol{H}^{2}(l)$ for each $l \in \gamma_{h, i j}$ and therefore we can apply standard interpolation estimates element-wise. We choose then $\boldsymbol{w}_{h, i}=\Pi_{i}^{*} \hat{\boldsymbol{u}}_{i 1} \times \Pi_{i}^{*} \hat{\boldsymbol{u}}_{i 2}$ 
and to obtain the desired result we note that

$$
\begin{gathered}
\left\|\mathcal{P}^{-1} \hat{\boldsymbol{u}}-\boldsymbol{w}_{h}\right\|_{\boldsymbol{H}_{\mathrm{div}}\left(\gamma_{h, i j}\right)}^{2}=\sum_{l \in \gamma_{h, i j}}\left\|\mathcal{P}^{-1} \hat{\boldsymbol{u}}_{i j}-\Pi_{i}^{*} \hat{\boldsymbol{u}}_{i j}\right\|_{\boldsymbol{H}_{\mathrm{div}}(l)}^{2}=\sum_{l \in \gamma_{h, i j}}\left\|\mathcal{P}^{-1} E_{i j}^{2} \hat{\boldsymbol{u}}_{i j}-\Pi_{i}^{*} \hat{\boldsymbol{u}}_{i j}\right\|_{\boldsymbol{H}_{\mathrm{div}}(l)}^{2} \\
\leq \sum_{l \in \gamma_{h, i}}\left\|\mathcal{P}^{-1} E_{i j}^{2} \hat{\boldsymbol{u}}_{i j}-\Pi_{i}^{*} \hat{\boldsymbol{u}}_{i j}\right\|_{\boldsymbol{H}_{\mathrm{div}}(l)}^{2} \leq C \sum_{l \in \gamma_{h, i}}|l|^{2}\left|\nabla_{\boldsymbol{\tau}_{h, i}} \cdot \mathcal{P}^{-1} E_{i j}^{2} \hat{\boldsymbol{u}}_{i j}\right|_{\boldsymbol{H}^{1}(l)}^{2} \\
\leq C h^{2} \sum_{l^{c} \in \gamma_{h, i}^{c}}\left|\nabla_{\boldsymbol{\tau}_{h, i}} \cdot E_{i j}^{2} \hat{\boldsymbol{u}}_{i j}\right|_{\boldsymbol{H}^{1}\left(l^{c}\right)}^{2} \\
\leq C h^{2}\left\|\hat{\boldsymbol{u}}_{i j}\right\|_{\boldsymbol{H}^{2}\left(\gamma_{i}\right)}^{2} \leq C h^{2}\left\|\hat{\boldsymbol{u}}_{i j}\right\|_{\boldsymbol{H}^{2}\left(\gamma_{i j}\right)}^{2} .
\end{gathered}
$$

We have exploited (5.4), standard interpolation error estimate for $\mathbb{R T}_{0}$ elements, as well as the properties of the extension operator $E_{i j}^{2}$, namely $\left.E_{i j}^{2} \hat{\boldsymbol{u}}_{i j}\right|_{\gamma_{i j}}=\hat{\boldsymbol{u}}_{i j}$ and $\left\|E_{i j}^{2} \hat{\boldsymbol{u}}_{i j}\right\|_{\boldsymbol{H}^{2}\left(\gamma_{i}\right)} \leq C\left\|\hat{\boldsymbol{u}}_{i j}\right\|_{\boldsymbol{H}^{2}\left(\gamma_{i j}\right)}$ (see [9]). We only need to control the jump term, where it is sufficient to exploit the trace inequality.

We finally obtain

$$
\inf _{\boldsymbol{w}_{h}^{c} \in \boldsymbol{W}_{h}^{c}}\left\|\hat{\boldsymbol{u}}-\boldsymbol{w}_{h}^{c}\right\|_{\boldsymbol{W}} \lesssim h|\hat{\boldsymbol{u}}|_{\mathcal{W}}
$$

We proceed analogously for the pressure term. We consider the transformation $\mathcal{P}$ which maps $\left(q_{11}, q_{12}, q_{21}, q_{22}, q_{I}\right) \in Q_{h}$ to $\left(\mathcal{P}_{1} q_{11}, \mathcal{P}_{1} q_{12}, \mathcal{P}_{2} q_{21}, \mathcal{P}_{2} q_{22}, q_{I}\right) \in Q_{h}^{c}$. We have thanks to Lemma 5.1

$$
\left\|\hat{p}-q_{h}^{c}\right\|_{Q} \lesssim\left\|\mathcal{P}^{-1} \hat{p}-q_{h}\right\|_{Q_{h}} .
$$

We choose $q_{h}=\left(q_{h, 11}, q_{h, 12}, q_{h, 21}, q_{h, 22}, q_{h, I}\right)$ by applying the extended $L^{2}$ interpolant $\pi_{i}^{*}$ defined in [15] on $\left(\mathcal{P}_{i}^{-1} q_{i 1}, \mathcal{P}_{i}^{-1} q_{i 2}\right)$, for $i=1,2$, while we set $q_{h, I}=q_{I}$. Using the result of the interpolation error for this extended interpolant we have

$$
\left\|\mathcal{P}^{-1} \hat{p}-q_{h}\right\|_{Q_{h}} \lesssim \sum_{i=1}^{2} \sum_{l_{h, i} \in \gamma_{h, i}}\left|l_{h, i}\right|\left|\mathcal{P}_{i}^{-1} \hat{p}\right|_{H^{1}\left(\gamma_{h, i}\right)} .
$$

We then apply (5.10) on each $\gamma_{h, i j}$ to map back on the curve $\gamma_{i j}$ and obtain the wanted result.

Remark 5.5. In the numerical setting we will solve the problem in the form given by (5.14). Note that, since we use $\mathbb{R T}_{0}$ finite elements we may replace the norm $L_{h}^{2}\left(\gamma_{h, i}\right)$ with the (simpler to compute) norm $L^{2}\left(\gamma_{h, i}\right)$. Indeed, since $\dot{D}=\mathcal{O}(h)$ by the application of the Strang Lemma to our problem we obtain a solution converging with the same order of convergence.

\section{Applicative examples}

We present some numerical experiments to validate the proposed reduced model and verify the theoretical results.

\subsection{Model error}

We start with an analysis to validate the reduced model presented in Section 3. We consider two rectilinear fractures $\gamma_{1}$ and $\gamma_{2}$ of thickness $d_{i}=0.005$ intersecting orthogonally. The fracture permeability are $K_{1, \tau}=1$ and $K_{2, \boldsymbol{\tau}}=10^{-2}$ respectively and in the intersection we have $\boldsymbol{K}_{I}=10^{-2} \boldsymbol{I}$. Thus, $\gamma_{2}$ acts as a barrier for the other fracture. The scalar source term is set to zero in both fractures and we take $\hat{\xi}_{0}=0.25$. We impose only essential boundary conditions, namely $g_{1,1}=0, g_{1,2}=1, g_{2,1}=-1$ and $g_{2,2}=1$. 

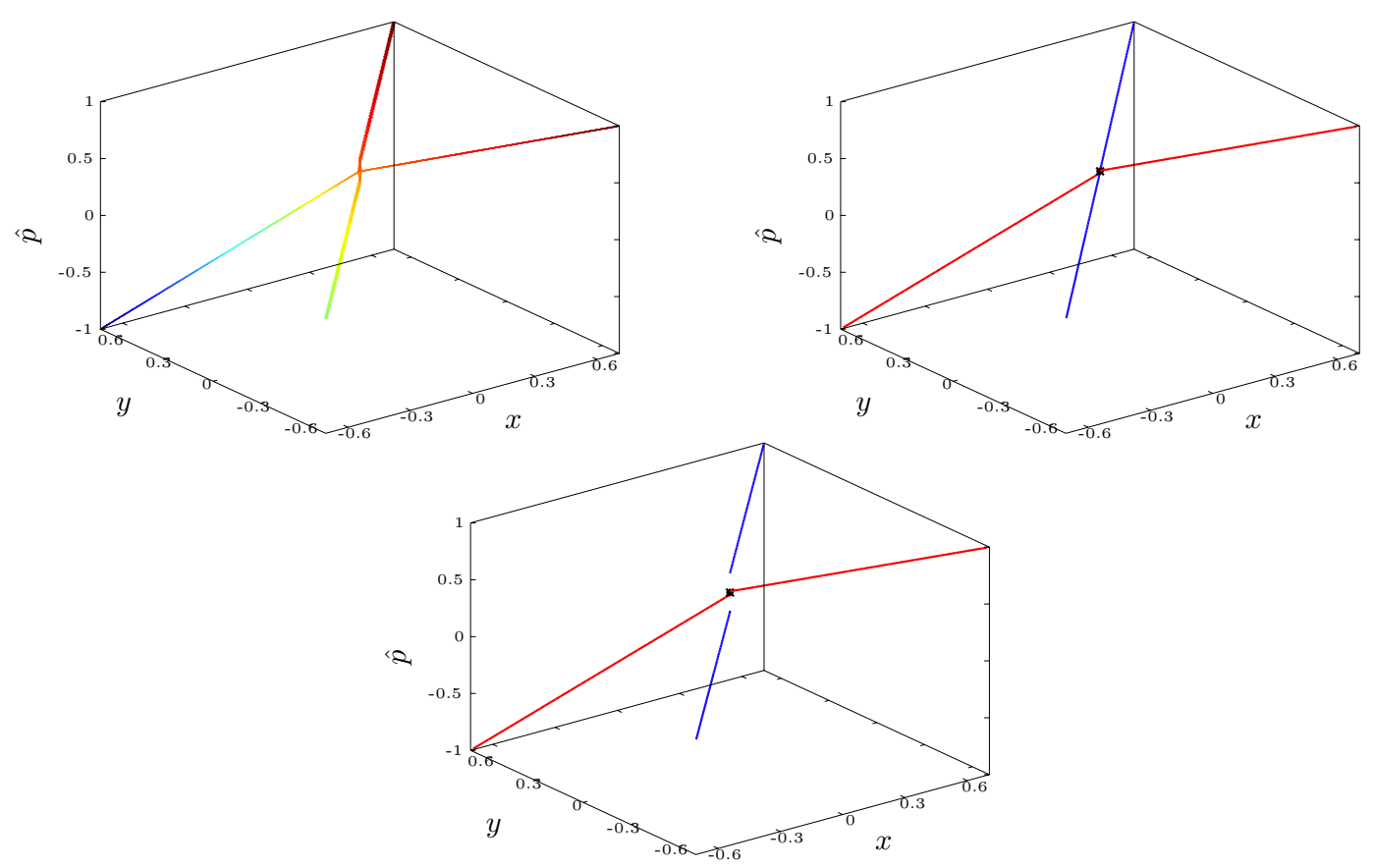

Figure 6 . In the top-left figure $p$ given by problem (2.3). In the top-right figure the solution of the reduced model (3.11a) with the interface conditions of $[4,6]$. At the bottom the solution of the reduced model (3.11). In the $1 D$ simulations $\gamma_{1}$ is colored in blue and $\gamma_{2}$ in red.

We want to compare the results obtained with the reduced model with a reference solution obtained solving the complete two-dimensional problem with a very fine two-dimensional grid of approximately $120 \times 10^{3}$ triangular elements.

We compare our reduced model with that proposed in $[4,6]$, where continuity of pressure is assumed at the interface. The results are represented in Figure 6. The solution of the two-dimensional problem is smooth in the intersection region but nevertheless exhibits a steep pressure gradient due to the low permeability imposed in $\Omega_{2}$ and in the intersection region. If we consider the reduced model with the coupling conditions presented in $[4,6]$ the solution cannot reproduce this behavior, while with the proposed conditions (3.11b) we are able to replace the pressure gradient of the $2 D$ solution with a correct pressure jump at the intersection and thus obtain the correct pressure gradient and flux in each branch of the fractures.

We now consider the behavior of our reduced model for different values of the parameters. To this purpose we compare the pressure of the fractures at the intersection point and the pressure in the intersection obtained solving the original problems (2.3a) and (2.3b) with the reduced pressures given by (3.2), (3.3). The solution of the two-dimensional problem is computed again with a fine grid and the computed pressure is averaged in the intersection region and on each edge of $I$ to obtain the values to compare with those produced by the reduced model.

We first consider the effect of the intersection angle. Let the two fractures have slope $\pm m$, respectively in the $x y$ plane. We set $K_{1, \tau}=1, K_{2, \boldsymbol{\tau}}=10^{-2}, \boldsymbol{K}_{I}=10^{-2} \boldsymbol{I}$ and $d_{i}=10^{-2}$. Table 1 shows the comparison of the pressures for different values of $m$. The relative error $\operatorname{err}_{\text {rel }}$ is computed as the ratio between the difference of the corresponding pressures and the maximum value of the pressure boundary conditions. We can see that the errors are rather small and independent on $m$. This indicates that reduced model is sound and capable of treating reasonably well intersections at different angle. 
TABle 1. Comparison of the model error for different values of the slope $m$. The twodimensional mesh is formed by $\sim 120 k$ triangles, while both the mono-dimensional meshes have $\sim 50$ segments.

\begin{tabular}{cccccccccc}
\cline { 2 - 9 } & \multicolumn{3}{c}{$|m|=1$} & \multicolumn{3}{c}{$|m|=10$} & \multicolumn{3}{c}{$|m|=0.1$} \\
\cline { 2 - 10 } & $2 D$ & $1 D$ & $e r r_{\text {rel }}$ & $2 D$ & $1 D$ & $e r r_{\text {rel }}$ & $2 D$ & $1 D$ & $e r r_{\text {rel }}$ \\
\hline$p_{I}$ & 0.494 & 0.493 & $0.1 \%$ & 0.495 & 0.493 & $0.2 \%$ & 0.495 & 0.493 & $0.2 \%$ \\
$\hat{p}_{1,1}$ & 0.366 & 0.327 & $3.9 \%$ & 0.324 & 0.331 & $0.7 \%$ & 0.314 & 0.324 & $1 \%$ \\
$\hat{p}_{1,2}$ & 0.624 & 0.663 & $3.9 \%$ & 0.666 & 0.660 & $0.6 \%$ & 0.676 & 0.666 & $1 \%$ \\
$\hat{p}_{2,1}$ & 0.492 & 0.478 & $1.4 \%$ & 0.639 & 0.642 & $0.3 \%$ & 0.337 & 0.317 & $2 \%$ \\
$\hat{p}_{2,2}$ & 0.496 & 0.498 & $0.2 \%$ & 0.351 & 0.334 & $1.7 \%$ & 0.652 & 0.658 & $0.6 \%$ \\
\hline
\end{tabular}

TABLE 2. Comparison of the model error for decreasing values of the thickness $d_{i}$. The spacing of the meshes are the same as in Table 1.

\begin{tabular}{cccccccccc} 
& \multicolumn{3}{c}{$d_{i}=0.01$} & \multicolumn{3}{c}{$d_{i}=0.005$} & \multicolumn{3}{c}{$d_{i}=0.0025$} \\
\cline { 2 - 10 } & $2 D$ & $1 D$ & $e r r_{\text {rel }}$ & $2 D$ & $1 D$ & $e r r_{\text {rel }}$ & $2 D$ & $1 D$ & $e r r_{\text {rel }}$ \\
\hline$p_{I}$ & 0.495 & 0.494 & $0.1 \%$ & 0.495 & 0.494 & $0.1 \%$ & 0.495 & 0.495 & - \\
$\hat{p}_{1,1}$ & 0.410 & 0.393 & $1.7 \%$ & 0.449 & 0.437 & $1.2 \%$ & 0.471 & 0.463 & $0.8 \%$ \\
$\hat{p}_{1,2}$ & 0.580 & 0.597 & $1.7 \%$ & 0.541 & 0.553 & $1.2 \%$ & 0.519 & 0.527 & $0.8 \%$ \\
$\hat{p}_{2,1}$ & 0.493 & 0.484 & $0.9 \%$ & 0.494 & 0.485 & $0.9 \%$ & 0.495 & 0.486 & $0.9 \%$ \\
$\hat{p}_{2,2}$ & 0.496 & 0.499 & $0.3 \%$ & 0.495 & 0.498 & $0.3 \%$ & 0.495 & 0.498 & $0.3 \%$ \\
\hline
\end{tabular}

TABLE 3. Comparison of the model error for different values of $\boldsymbol{K}_{I}$. The spacing of the meshes are the same as in Table 1.

\begin{tabular}{ccccccccccccc} 
& \multicolumn{4}{c}{$\boldsymbol{K}_{I}=K_{1, \boldsymbol{\tau}} \boldsymbol{I}$} & \multicolumn{3}{c}{$\boldsymbol{K}_{I}=K_{2, \boldsymbol{\tau}} \boldsymbol{I}$} & \multicolumn{3}{c}{$\boldsymbol{K}_{I}=\boldsymbol{K}_{H}$} & \multicolumn{3}{c}{$\boldsymbol{K}_{I}=\boldsymbol{K}_{T}$} \\
\cline { 2 - 13 } & $2 D$ & $1 D$ & $e r r_{\mathrm{rel}}$ & $2 D$ & $1 D$ & $e r r_{\mathrm{rel}}$ & $2 D$ & $1 D$ & $e r r_{\mathrm{rel}}$ & $2 D$ & $1 D$ & $e r r_{\mathrm{rel}}$ \\
\hline$p_{I}$ & 0.500 & 0.500 & - & 0.499 & 0.497 & $0.2 \%$ & 0.499 & 0.499 & - & 0.500 & 0.500 & - \\
$\hat{p}_{1,1}$ & 0.497 & 0.493 & $0.4 \%$ & 0.019 & 0.010 & $0.9 \%$ & 0.028 & 0.019 & $0.9 \%$ & 0.497 & 0.505 & $0.8 \%$ \\
$\hat{p}_{1,2}$ & 0.502 & 0.507 & $0.5 \%$ & 0.981 & 0.990 & $0.9 \%$ & 0.972 & 0.981 & $0.9 \%$ & 0.502 & 0.485 & $1.7 \%$ \\
$\hat{p}_{2,1}$ & 0.500 & 0.485 & $1.5 \%$ & 0.497 & 0.483 & $1.4 \%$ & 0.498 & 0.488 & $1 \%$ & 0.500 & 0.505 & $0.5 \%$ \\
$\hat{p}_{2,2}$ & 0.500 & 0.505 & $0.5 \%$ & 0.500 & 0.502 & $0.2 \%$ & 0.500 & 0.502 & $0.2 \%$ & 0.500 & 0.505 & $0.5 \%$ \\
\hline
\end{tabular}

Table 2 shows instead the relative errors when the thickness of the fractures decreases. In this case we have taken $\boldsymbol{K}_{I}=50.5^{-1} \boldsymbol{I}$, i.e. the harmonic mean of the $K_{i, \boldsymbol{\tau}}$, and $|m|=1$. Also in this case the errors are rather small and, as we expected, they decrease when the thicknesses decrease. Even if this is not a rigorous analysis of the model error, it gives numerical evidence of its asymptotic behavior with respect to the fracture thickness.

Finally we address some different choices to prescribe the permeability in the intersection region. The choice should of course be driven by physical arguments. For instance, if we assume that $\gamma_{1}$ is "younger" then $\gamma_{2}$, i.e. it was generated after $\gamma_{2}$, than $\boldsymbol{K}_{I}$ should be equal to the $K_{1, \boldsymbol{\tau}}$. Conversely, if we assume that $\gamma_{2}$ is "younger" then $\gamma_{1}$, we should set $\boldsymbol{K}_{I}=K_{2, \boldsymbol{\tau}}$. Alternatively we can impose a tensor $\boldsymbol{K}_{H}=K_{H} \boldsymbol{I}$ that is the harmonic mean of $K_{i, \boldsymbol{\tau}}$, if we suppose that the material of each $\gamma_{i}$ has been mixed in the intersection region $I$. Finally we can impose to $\boldsymbol{K}_{I}$, in the direction aligned to each $\gamma_{i}$, the value $K_{i, \boldsymbol{\tau}}$ obtaining a non-isotropic tensor $\boldsymbol{K}_{T}$.

In Table 3 we compare the four choices for a system of two orthogonal fractures of thickness $d_{i}=0.01$ and permeability $K_{1, \tau}=1, K_{2, \tau}=10^{-4}$. We can observe that, for $\boldsymbol{K}_{I}=K_{2, \boldsymbol{\tau}}$ and $\boldsymbol{K}_{I}=\boldsymbol{K}_{H}$ the solution exhibits a pressure jump along $\gamma_{2}$ while in the other cases the solution is almost continuous. In all cases the relative error is of the order of $1 \%$ or less proving the effectiveness of the reduced model with the new coupling conditions. 

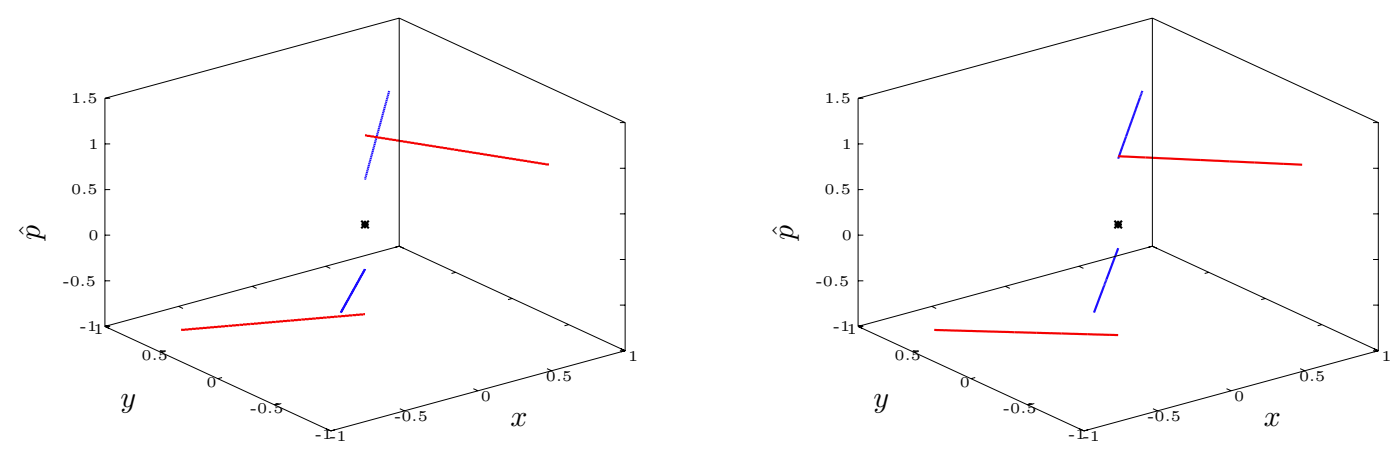

Figure 7 . In the left image $\hat{\xi}_{0}$ is such that the maximum principle is not fulfilled, while in the right is fulfilled.

TABle 4. Top values of $\max _{i} \hat{p}_{i}$ for different $\hat{\xi}_{0}$ in the orthogonal case, bottom for the nonorthogonal case. In bold the values of $\hat{\xi}_{0}$ and of the maximum pressure that fulfill the maximum principle.

\begin{tabular}{|c|c|c|c|c|c|}
\hline$\hat{\xi}_{0}$ & 0 & 0.05 & 0.1 & $\mathbf{0 . 1 5}$ & $\mathbf{0 . 2}$ \\
\hline $\max _{i} \hat{p}_{i}$ & 1.228 & 1.003 & $\mathbf{0 . 9 9 2}$ & $\mathbf{0 . 9 8 8}$ & $\mathbf{0 . 9 8 6}$ \\
\hline \hline$\hat{\xi}_{0}$ & 0 & 0.0025 & $\mathbf{0 . 0 0 5}$ & 0.0075 & 0.01 \\
\hline $\max _{i} \hat{p}_{i}$ & 1.116 & 1.033 & $\mathbf{0 . 9 9 3}$ & 1.018 & 1.035 \\
\hline
\end{tabular}

\subsection{Maximum principle}

We want to verify, with numerical experiments, the bounds derived in Theorem 5.3 for the parameter $\hat{\xi}_{0}$ that ensure the fulfillment of the maximum principle.

Let us consider two fractures of the same length $L=1$ and thickness $d_{i}=0.01$ that intersect orthogonally. We set $\hat{\eta}_{i}=1$ while in the intersection point we consider an isotropic permeability tensor $\boldsymbol{K}_{I}=10^{-4} \boldsymbol{I}$. We impose pressure as a boundary condition on all four end points, in particular we set $g_{i, 1}=0$ and $g_{i, 2}=1$ for $i=1,2$. For this configuration, according to Theorem 5.3, the maximum principle is satisfied if $\hat{\xi}_{0} \geq 1 / 8$. Figure 7 shows the solution we obtained with $\hat{\xi}_{0}=0$, a value that does not satisfy the hypotheses: it is clear that the maximum principle is violated, indeed the pressure inside the domain exceeds 1 which is the maximum at the boundary. In the same figure we represent the solution obtained with $\hat{\xi}_{0}=1 / 8$, which satisfies the maximum principle, as indicated by the theory.

In the first part of Table 4 we report the maximum value of pressure in the domain for different $\hat{\xi}_{0}$ to prove that the maximum principle is violated for some values of the parameter outside the theoretical bounds.

We then consider two fractures that intersect forming a small angle of $0.2 \mathrm{rad}$. In this case the bounds on $\hat{\xi}_{0}$ are $4.934 \times 10^{-3} \leq \hat{\xi}_{0} \leq 5.034 \times 10^{-3}$. We report in the second part of Table 4 the maximum pressure in the domain for different $\hat{\xi}_{0}$ : it can be observed that 0.005 is indeed the only value for which the solution fulfills the maximum principle. This numerical experiment points out that for small intersection angles the bounds are non only stricter but also sharper than in the case of orthogonal fractures. We recall that the bounds represent only a sufficient condition.

\subsection{Convergence rates}

Let us consider two intersecting fractures described by equations

$$
\gamma_{1}=\{(x, y): y=x\} \quad \text { and } \quad \gamma_{2}=\{(x, y): y=1-x\}, \quad x \in\left[-\frac{\sqrt{2}}{2}, \frac{\sqrt{2}}{2}\right] .
$$




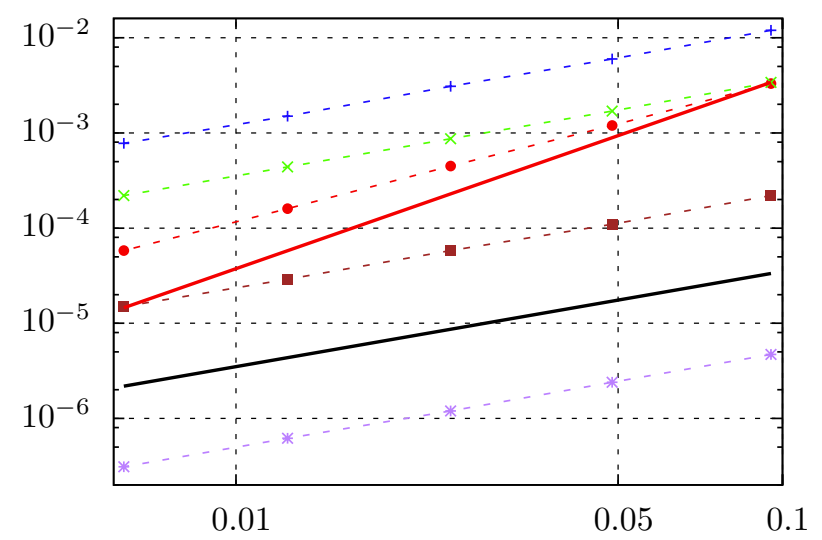

$$
\begin{array}{ll}
\left\|\hat{p}_{h}-\hat{p}\right\| & \ldots \cdot- \\
\left|\hat{p}_{I, h}-\hat{p}_{I}\right| & \ldots+- \\
\left\|\hat{\boldsymbol{u}}_{h}-\hat{\boldsymbol{u}}\right\| & \ldots *- \\
\left\|\nabla_{\boldsymbol{\tau}} \cdot\left(\hat{\boldsymbol{u}}_{h}-\hat{\boldsymbol{u}}\right)\right\| & \ldots \ldots- \\
\left|\llbracket \hat{\boldsymbol{u}}_{h}-\hat{\boldsymbol{u}} \rrbracket_{i_{p}} \cdot \boldsymbol{\tau}\right| & \ldots-\cdots \\
\text { reference } \mathcal{O}(h) & \\
\text { reference } \mathcal{O}\left(h^{2}\right) &
\end{array}
$$

Figure 8. Convergence rates for each pieces of the norm $\boldsymbol{W}$ and $Q$. In the legend we have indicated by $\|\cdot\|$ the $L^{2}$ norm.

The permeability of the fractures is $K_{i, \boldsymbol{\tau}}=1$ while $\boldsymbol{K}_{I}=0.01 \boldsymbol{I}$. Both fractures have thickness $d_{i}=0.01$, the boundary conditions are $\boldsymbol{g}=[0,1,0,1]$ and the source term is set to $f_{1}=0.01$ only in $\gamma_{1}$ for $x<0$. Finally choosing $\xi_{0}=1 / 4$, the exact solution is

$$
\begin{gathered}
\hat{p}_{1}(s)=\left\{\begin{array}{ll}
-\frac{s^{2}}{2}+\frac{13}{12} s & s \in[0,1) \\
\frac{1}{4} s+1 & s \in(1,2]
\end{array}, \quad \hat{p}_{2}(s)= \begin{cases}\frac{5}{12} s & s \in[0,1) \\
\frac{1}{4} s+1 & s \in(1,2]\end{cases} \right. \\
\hat{\boldsymbol{u}}_{1}(s)=\left\{\begin{array}{ll}
s-\frac{13}{12} & s \in[0,1) \\
-\frac{1}{4} & s \in(1,2]
\end{array}, \quad \hat{\boldsymbol{u}}_{2}(s)= \begin{cases}\frac{5}{12} & s \in[0,1) \\
-\frac{1}{4} & s \in(1,2]\end{cases} \right.
\end{gathered}
$$

and $\hat{p}_{I}=5 / 8$. Figure 8 shows the errors computed with decreasing grid spacings. The numerical results are in good agreement with Theorem 5.4. Moreover there are a numerical evidences that in all the examples analyzed, like the ones presented, the pressure error decay better then $\mathcal{O}(h)$. A possible motivation is the regularity of the pressure, separately, in each sub-division of the computational domain. In both example we have $\left\|\hat{p}_{h}-\hat{p}\right\| \simeq \mathcal{O}\left(h^{1.5}\right)$. However a deeper explanation of this fact is out of the scope of the present work.

Let us now consider the case of curved fractures to evaluate the error associated with the approximation of geometry as piecewise linear. The two fractures are now described by the following equations

$$
\gamma_{1}=\{(x, y): x=0, y=1+2 \theta\} \quad \text { and } \quad \gamma_{2}=\{(x, y): x=\sin (\pi \theta), y=1+\cos (\pi \theta)\}, \quad \theta \in[-1,0] .
$$

We impose the same permeability, thicknesses, source term and boundary conditions as in the previous case: since the arc length of the four branches is the same we obtain the same exact solution. The errors obtained for different grid spacings are reported in Figure 9. It can be observed that the error decreases linearly as in the case of straight fractures and the absolute values are comparable, thus, if the grid size is small enough compared to the fracture curvature, the approximation of geometry does not affect the quality of the numerical solution.

\subsection{Networks of fractures}

As already mentioned the method proposed in this paper can be applied to networks composed by an arbitrary number of fractures. Let us consider a set of three fractures $\gamma_{1,2,3}$ characterized by the same permeability. 


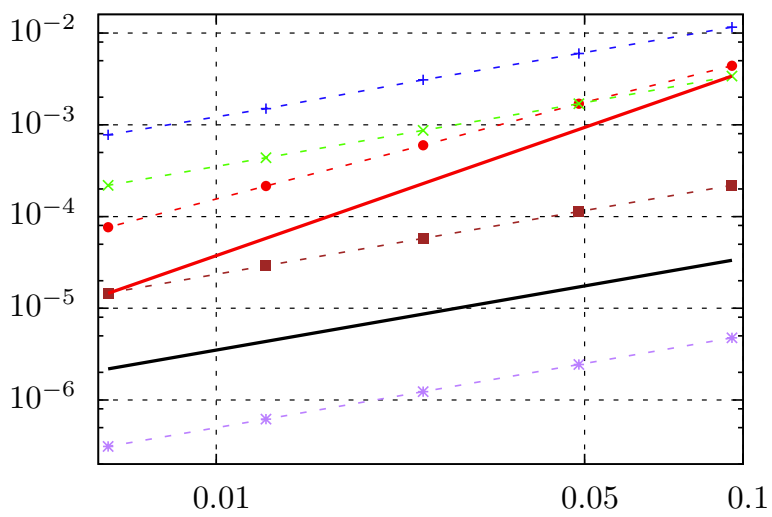

$$
\begin{array}{ll}
\left\|\hat{p}_{h}-\hat{p}\right\| & \ldots \cdot- \\
\left|\hat{p}_{I, h}-\hat{p}_{I}\right| & \ldots+\cdots \\
\left\|\hat{\boldsymbol{u}}_{h}-\hat{\boldsymbol{u}}\right\| & \ldots *- \\
\left\|\nabla_{\boldsymbol{\tau}} \cdot\left(\hat{\boldsymbol{u}}_{h}-\hat{\boldsymbol{u}}\right)\right\| & \ldots *- \\
\left|\llbracket \hat{\boldsymbol{u}}_{\boldsymbol{h}}-\hat{\boldsymbol{u}} \rrbracket_{\boldsymbol{i}_{p}} \cdot \boldsymbol{\tau}\right| & \ldots-\cdots \\
\text { reference } \mathcal{O}(h) & - \\
\text { reference } \mathcal{O}\left(h^{2}\right) &
\end{array}
$$

FiguRE 9. Convergence rates for the curve case.
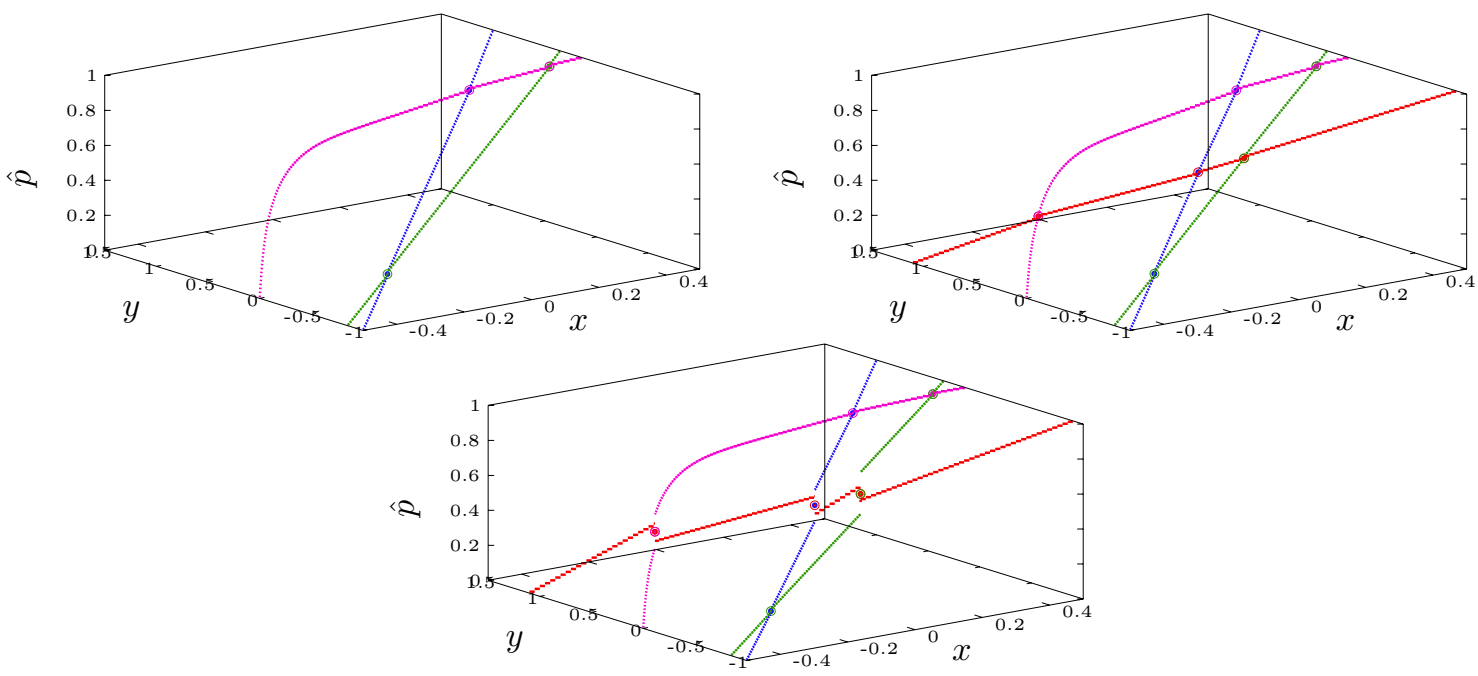

FiguRE 10. Top-left: $\hat{p}_{i}$ without $\gamma_{4}$. Top-right and bottom: $\hat{p}_{i}$ with the classical conditions and our model, respectively.

Imposing $g_{i, 1}=0$ and $g_{i, 2}=1$ for all $i$ we obtain the pressure distribution reported in Figure 10. We now insert a new fracture $\gamma_{4}$ with lower permeability, and, following the considerations of Section 6.1, we impose in all the intersection with $\gamma_{4}$ the permeability of this latter. As shown in Figure 10 the solution obtained with the classical model $[4,6]$ is everywhere continuous while the new coupling conditions allow us to mimic the blocking nature of $\gamma_{4}$.

In realistic applications fractured porous media are often characterized by the density and orientation of the fractures rather than by detailed information on the geometry and properties of the single fracture. In this second test case we consider a grid of $N_{H}$ horizontal fractures and $N_{V}$ fractures that form a variable angle $\theta$ with the horizontal ones. We impose homogeneous boundary conditions for the pressure on all tips except for one where we set $g_{1,2}=1$ as shown in Figure 11 left. Figure 11 right shows the resulting pressure field for $N_{H}=N_{V}=5$ and $\theta=80^{\circ}$. Thanks to the efficiency of the reduced one-dimensional model we are able to analyze different configuration with a low computational cost. Figure 12 shows the value of the pressure in the center of the network for different orientations of the fractures, i.e. different angles $\theta$, and for increasing density 

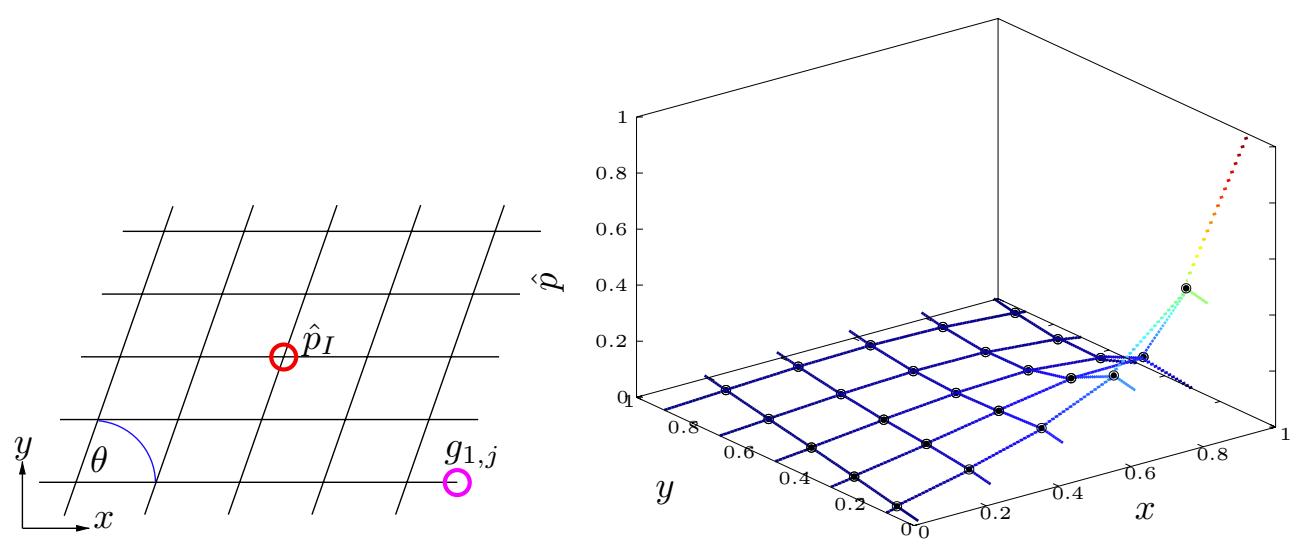

FigURE 11. Left grid of fractures with the interesting point. Right simulation of ten fractures with $\theta=80^{\circ}$.

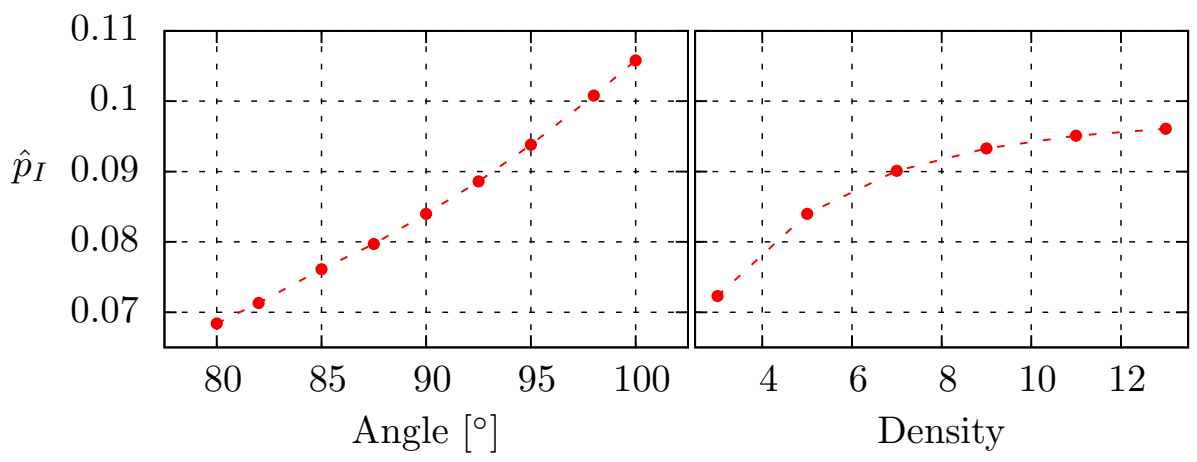

FIGURE 12. In the left $\hat{p}_{I}$ for different values of the angle between the fractures. In the right $\hat{p}_{I}$ increasing the number of the fractures.

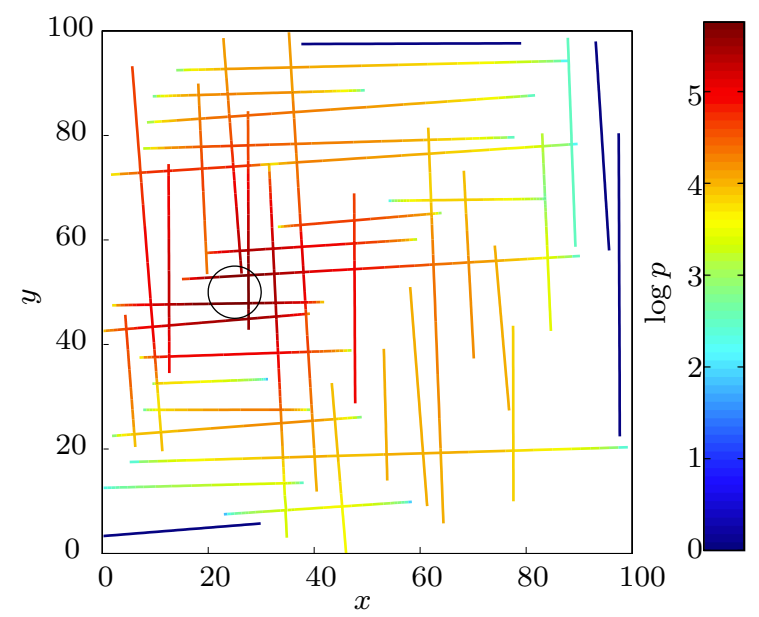

FiguRE 13. Distribution of the $\log p$ in the fracture network. The area interested by the source term is highlighted. 
of the vertical fractures in the orthogonal case. We can observe that as we increase the number of fractures, thus the transmissibility of the network, the pressure in the central point tends to an asymptotic value.

Finally, we consider a more realistic test case where the fracture distribution is generated randomly starting from a grid of horizontal and vertical fractures of various length and perturbing the angles. We consider 40 fractures of width $d=1 \mathrm{~mm}$ and permeability of $1 \mathrm{mD}\left(10^{-12} \mathrm{~Pa} \mathrm{~s}\right)$. The resulting network is approximately $100 \mathrm{~m}$ wide. We point out that since the fracture position and orientation is random some may not be connected with the rest of the network. No flux boundary conditions are prescribed at the tips and a source term $\hat{f}=10^{-8} \mathrm{~m} / \mathrm{s}$ is imposed in the circular region of radius $R=5 \mathrm{~m}$ represented in Figure 13, such that only a few fractures are affected. The resulting pressure field is represented in Figure 13 and reflects the connectivity of the network.

\section{Conclusions}

In this paper we derived and analyzed a reduced model for flow in a network of fractures. The derivation is similar to that given in $[15,27]$, yet here we propose new coupling conditions to handle in a more realistic way the intersecting fractures. These conditions take into account the intersection angle and, by allowing a discontinuous pressure at the interface they are capable of giving accurate results also in the case where the permeability of the fractures are very different. This is not the case for the coupling conditions in $[5,6]$. This can be relevant to applications since a fracture may sometimes act as a barrier or a preferential path.

Well posedness analysis has been given for both the continuous and discrete problem and numerical experiments have been performed to validate the theoretical results of convergence and positivity. The comparison with two dimensional simulations on refined grids proved that the new coupling conditions give reasonably accurate results, and they perform better than the classical one in the case of impermeable fractures. We have also shown how the model can be used to simulate more realistic configurations with the presence of several intersecting fractures.

Further developments will consist in extending the analysis to the coupling between the network and the surrounding porous medium introduced in [18], to obtain a complete framework for the simulation of monophase flow in presence of an arbitrary set of fractures.

We have dealt only with two dimensional problems. However the derivation here presented forms the basis for a similar reduced model in a three dimensional setting. The main difficulty in the extension to three dimensional problems is that the interface condition is not anymore an algebraic one, but it involves the interaction with a one-dimensional model that describes the flow along the intersection. This matter is the subject of ongoing work. Two dimensional simulations have however, already an applicative relevance. They may be used to better understand the behavior of the flow in the presence of fractures and drive, for instance, upscaling techniques.

Acknowledgements. This work has been supported by ENI s.p.a. The first and third authors also acknowledge the support of MIUR through the PRIN09 Project No. 2009Y4RC3B 001.

\section{REFERENCES}

[1] R.T. Adams, Sobolev Spaces, vol. 65. Pure and Applied Mathematics. Academic Press (1975).

[2] P.M. Adler and J.-F. Thovert, Fractures and fracture networks. Springer (1999).

[3] P.M. Adler, J.-F. Thovert and V.V. Mourzenko, Fractured porous media. Oxford University Press (2012).

[4] C. Alboin, J. Jaffré, J.E. Roberts and C. Serres, Modeling fractures as interfaces for flow and transport in porous media, in Fluid flow and transport in porous media: mathematical and numerical treatment (South Hadley, MA, 2001), vol. 295. Contemp. Math.. Amer. Math. Soc. Providence, RI (2002) 13-24.

[5] C. Alboin, J. Jaffré, J.E. Roberts, X. Wang and C. Serres. Domain decomposition for some transmission problems in flow in porous media, vol. 552. Lect. Notes Phys. Springer, Berlin (2000) 22-34.

[6] L. Amir, M. Kern, V. Martin and J.E. Roberts, Décomposition de domaine et préconditionnement pour un modèle 3D en milieu poreux fracturé, in Proc. of JANO 8, 8th Conf. Numer. Anal. Optim. (2005).

[7] P. Angot, F. Boyer and F. Hubert, Asymptotic and numerical modelling of flows in fractured porous media. ESAIM: M2AN 43 (2009) 239-275.

[8] J. Bear, C.-F. Tsang and G. de Marsily, Flow and contaminant transport in fractured rock. Academic Press, San Diego (1993). 
[9] R. Becker, E. Burman and P. Hansbo, A Nitsche extended finite element method for incompressible elasticity with discontinuous modulus of elasticity. Comput. Methods Appl. Mech. Engrg. 198 (2009) 3352-3360.

[10] B. Berkowitz, Characterizing flow and transport in fractured geological media: A review. Adv. Water Resources 25 (2002) 861-884.

[11] S. Berrone, S. Pieraccini and S. Scialò, On simulations of discrete fracture network flows with an optimization-based extended finite element method. SIAM J. Sci. Comput. 35 (2013) 908-935.

[12] S. Berrone, S. Pieraccini and S. Scialò, A PDE-constrained optimization formulation for discrete fracture network flows. SIAM J. Sci. Comput. 35 (2013).

[13] H. Brezis, Functional analysis, Sobolev spaces and partial differential equations. Springer (2010).

[14] F. Brezzi and M. Fortin, Mixed and Hybrid Finite Element Methods, vol. 15. Comput. Math. Springer Verlag, Berlin (1991).

[15] C. D'Angelo and A. Scotti, A mixed finite element method for Darcy flow in fractured porous media with non-matching grids. ESAIM: M2AN 46 (2012) 465-489.

[16] A. Ern and J.L. Guermond, Theory and practice of finite elements. Appl. Math. Sci. Springer (2004).

[17] B. Faybishenko, P.A. Witherspoon and S.M. Benson, Dynamics of fluids in fractured rock, vol. 122. Geophysical monographs. American geophysical union (2000).

[18] A. Fumagalli, Numerical Modelling of Flows in Fractured Porous Media by the XFEM Method. Ph.D. thesis, Politecnico di Milano (2012).

[19] A. Fumagalli and A. Scotti, A numerical method for two-phase ow in fractured porous media with non-matching grids, in vol. 62 of Adv. Water Resources (2013) 454-464.

[20] A. Fumagalli and A. Scotti, A reduced model for flow and transport in fractured porous media with non-matching grids, Numer. Math. Advanced Applications 2011. Edited by A. Cangiani, R.L. Davidchack, E. Georgoulis, A.N. Gorban, J. Levesley and M.V. Tretyakov. Springer Berlin, Heidelberg (2013) 499-507.

[21] B. Gong, G. Qin, C. Douglas and S. Yuan, Detailed modeling of the complex fracture network of shale gas reservoirs. SPE Reservoir Evaluation and Engrg. (2011).

[22] A. Hansbo and P. Hansbo, A finite element method for the simulation of strong and weak discontinuities in solid mechanics. Comput. Methods Appl. Mech. Engrg. 193 (2004) 3523-3540.

[23] M. Hussein and D. Roussos, Discretizing two-dimensional complex fractured fields for incompressible two-phase flow. Int. J. Numer. Methods Fluids (2009).

[24] J. Jaffré, V. Martin and J.E. Roberts, Generalized cell-centered finite volume methods for flow in porous media with faults, in Finite volumes for complex applications III (Porquerolles, 2002). Hermes Sci. Publ., Paris (2002) 343-350.

[25] J. Jaffré, M. Mnejja and J.E. Roberts, A discrete fracture model for two-phase flow with matrix-fracture interaction. Procedia Comput. Sci. 4 (2011) 967-973.

[26] M. Karimi-Fard, L.J. Durlofsky and K. Aziz, An Efficient Discrete-Fracture Model Applicable for General-Purpose Reservoir Simulators. SPE J. 9 (2004) 227-236.

[27] V. Martin, J. Jaffré and J.E. Roberts, Modeling fractures and barriers as interfaces for flow in porous media. SIAM J. Sci. Comput. 26 (2005) 1667-1691.

[28] H. Mustapha, A new approach to simulating flow in discrete fracture networks with an optimized mesh. SIAM J. Sci. Comput. 29 (2007) 1439-1459.

[29] A. Quarteroni and A. Valli, Numerical approximation of partial differential equations, vol. 23. Springer Ser. Comput. Math. Springer-Verlag, Berlin (1994).

[30] M. Sahimi, Flow and transport in porous media and fractured rock. Wiley-VCH, Weinheim (2011). 